\title{
High-throughput approaches towards the definitive identification of pharmaceutical drug metabolites. 2. An example of how unexpected dissociation behaviour could preclude correct assignment of sites of metabolism
}

Stephen W. Holman, Patricia Wright and G. John Langley

School of Chemistry, University of Southampton, Southampton UK SO17 1BJ. Email: gjl@soton.ac.uk

Please cite this paper as:

Rapid Communications in Mass Spectrometry, 2009, 2017-2025

The publisher's version of this paper is available here: http://dx.doi.org/10.1002/rcm.4103

\section{Related articles by Dr John Langley can be found below:}

A. Galezowska, M.W. Harrison, J.M. Herniman and G.J. Langley "A Predictive Science Approach: An Understanding of DFT Applications to the Mass Spectrometry of Small Molecules." Rapid Commun.Mass Spectrom. 2013, 27, 964-970. (DOI: 10.1002/rcm.6536)

S.W. Holman, P. Wright and G.J. Langley, "The low-energy collision-induced dissociation product ion spectra of protonated beta-blockers reveal an analogy to fragmentation under electron ionisation conditions", J. Mass Spectrom. 2011, 1182-1185. DOI 10.1002/jms.2006

S.W. Holman, P. Wright and G.J. Langley, "Rapid Methodology for the Characterization of Dialkyl Tertiary Amine- $N$-Oxide Metabolites Using Structurally Dependent Dissociation Pathways and Reconstructed Ion Current Chromatograms", Anal. Chem. 2010, 82 (6), 2347-2354. DOI: 10.1021/ac902540b.

S.W. Holman, P. Wright, N.J. Wells and G.J. Langley, "Evidence for site-specific intra-ionic hydrogen/deuterium exchange in the low-energy collision-induced dissociation product ion spectra of protonated small molecules generated by electrospray ionisation" J. Mass Spectrom. 2010, 45, 347-357. DOI: 10.1002/jms.1714.

S.W. Holman, P. Wright, and G.J. Langley, "High-throughput approaches towards the definitive identification of pharmaceutical drug metabolites. 1. Evidence for an ortho effect on the fragmentation of 4-benzenesulfinyl-3-methylphenylamine using electrospray ionisation-mass spectrometry." Rapid Commun. Mass Spectrom. 2008, 22, 2355-2365. DOI: 10.1002/rcm

K. Klagkou, F.S. Pullen, M.E. Harrison, A. Organ, A. Firth and G.J. "Fragmentation pathways of sulphonamides under electrospray tandem mass spectrometric conditions" Rapid Commun. Mass Spectrom., 2003, 17, 2373-2379. DOI: 10.1002/rcm.1201 
High-throughput approaches towards the definitive identification of pharmaceutical drug metabolites. 2 . An example of how unexpected dissociation behaviour could preclude correct assignment of sites of metabolism

\author{
Stephen W. Holman ${ }^{1}$, Patricia Wright ${ }^{2}$ and G. John Langley ${ }^{1 *}$ \\ ${ }^{1}$ School of Chemistry, University of Southampton, Southampton, SO17 1BJ, UK \\ ${ }^{2}$ Department of Pharmacokinetics, Dynamics and Metabolism, Pfizer Global Research and \\ Development, Ramsgate Road, Sandwich, CT13 9NJ, UK
}

Correspondence to: G. J. Langley, School of Chemistry, University of Southampton, Southampton, SO17 1BJ, UK,

E-mail: g.j.langley@soton.ac.uk

Tel: +44 2380593320

Fax: +44 2380597641 


\begin{abstract}
S-oxidation is a common metabolic route for sulphur containing compounds. When studying the fragmentation of some chemically synthesised sulphoxides, two unexpected losses of $62 \mathrm{~m} / \mathrm{z}$ units were observed in the collision-induced dissociation (CID) product ion spectrum protonated 3-dimethylaminomethyl-4-(4-methanesulfinyl-3-methyl-phenoxy)benzenesulfonamide. A single loss was initially assigned using the low resolution product ion spectrum, acquired by electrospray ionisation-quadrupole ion trap-mass spectrometry (ESIQIT-MS), as methanethial, S-oxide with the dissociation proceeding via a charge-remote, four-centred rearrangement. This assignment was consistent with well-documented hydrogen rearrangements in the literature. Further, the loss was not observed for the parent compound. Thus, it was inferred that the site of metabolism was involved in the dissociation and the attractive nature of the four-centred rearrangement meant that the loss of methanethial, $S$ oxide was a logical assignment. However, deuterium labelling experiments and accurate mass measurements, performed using electrospray ionisation-Fourier Transform Ion Cyclotron Resonance-mass spectrometry (ESI-FTICR-MS), showed that two distinct losses of $62 \mathrm{~m} / \mathrm{z}$ units occur, neither of which was of that initially hypothesised from the low resolution product ion spectrum of the protonated molecule. Mechanisms consistent with the experimental findings are postulated. An $\mathrm{MS}^{3}$ spectrum of the fully exchanged, deuterated species supported the proposed mechanisms by suggesting that 3-dimethylaminomethyl-4-(4methanesulfinyl-3-methyl-phenoxy)-benzenesulfonamide has multiple sites of protonation in the gas phase. The planar structures of the posited product ions are likely to provide the driving force for the rearrangements. The relevance of the observations with regards to pharmaceutical drug metabolite identification are discussed.
\end{abstract}

Keywords: Collision-induced dissociation; Fragmentation; Drug metabolite identification; Drug discovery; H/D exchange.

\title{
Introduction
}

Identification of the metabolites of pharmaceutical compounds is a key stage of drug discovery. Sites on the novel chemical entity prone to biotransformation are identified, thus allowing the pharmacokinetic behaviour of the molecule to be improved through alteration of the compound's structure. ${ }^{1,2}$ Active metabolites, i.e. those possessing pharmacological activity similar, synergistic or inhibitory to that of the parent compound, can be identified to rationalise pharmacodynamic results. ${ }^{3,4}$ Additionally, novel chemical entities that form 
reactive metabolites, resulting in toxicity, can be identified and either modified or eliminated to prevent failure of the drug candidate in the development stage. ${ }^{5}$

During early drug discovery metabolite identification studies, limited amounts of chemical bulk and the need for liquid chromatographic separation may prohibit the routine acquisition of nuclear magnetic resonance (NMR) spectra for definitive structural elucidation. Hence, mass spectrometry is routinely used to assign the sites of metabolism of pharmaceutical drug compounds. The principal method involves comparing the collision-induced dissociation (CID) product ion spectrum of the parent compound with that of the metabolite and looking for mass shifts of the major peaks in these spectra to determine which part of the molecule has been altered i.e. the Shift technique. ${ }^{6}$ However, unambiguous assignments by this approach are frequently not possible, particularly for oxidised metabolites where the biotransformation can take place at carbon, nitrogen or sulfur atoms; all common constituents of pharmaceutical compounds. ${ }^{7}$ Furthermore, the high-throughput nature of modern drug discovery means that multiple mass spectrometric-related structural elucidation experiments e.g. deuterium labelling, derivatisation etc., are rarely performed. Thus, there is a risk that incorrect assignment of sites of metabolism could arise. This is a particular likelihood given that the CID processes for molecules of this type are still not fully understood, with unexpected rearrangements under CID conditions being previously reported in the literature for both small and peptidic molecules. ${ }^{8-11}$ Herein, a further example of unanticipated dissociation behaviour, observed for the model sulfoxide detailed here, demonstrates how incorrect assignment of the sites of metabolism of a novel chemical entity could occur if a rigorous analytical approach is not applied.

\section{Experimental}

\section{Chemicals}

3-dimethylaminomethyl-4-(4-methanesulfinyl-3-methyl-phenoxy)-benzenesulfonamide

and 3-dimethyl- ${ }^{2} \mathrm{H}_{6}$-aminomethyl-4-(4-methanesulfinyl-3-methyl-phenoxy)benzenesulfonamide (2) were synthesised by Pfizer Global Research and Development (Sandwich, UK). Reserpine was purchased from Sigma-Aldrich Company Limited (Gillingham, Dorset, UK). Solutions were prepared in LC-MS grade methanol and analytical 
grade methanoic acid [99.9:0.1, v/v] (Fisher Scientific UK Limited, Loughborough, UK). Solutions of $\mathbf{1}$ and 2 were prepared at $10 \mu \mathrm{g} \mathrm{m}^{-1}$ for the quadrupole ion trap mass spectrometry (QIT-MS) experiments. Further solutions of 1 were prepared at $1 \mu \mathrm{g} \mathrm{mL}{ }^{-1}$ for the Fourier transform ion cyclotron resonance-mass spectrometry (FT-ICR-MS) experiments and $10 \mu \mathrm{g} \mathrm{mL}^{-1}$ in $>99.5 \%$ deuterated methanol and $99.5 \%$ deuterated ethanoic acid [99:1, v/v] (Apollo Scientific Limited, Stockport, UK) for hydrogen/deuterium (H/D) exchange experiments. All chemicals were used without further purification.

\section{Instrumental}

Product ion spectra of either the protonated or fully, exchanged deuterated molecule for each compound were acquired using a LCQ Classic QIT mass spectrometer (Thermo Fisher Scientific, San Jose, CA, USA). Positive ion electrospray (ESI) was performed by infusing the solutions directly into the source at a constant flow rate of $3 \mu \mathrm{L} \mathrm{min}^{-1}$. Nitrogen was used as the sheath and auxiliary gas. The source conditions were; sheath gas, 35 arbitrary units; auxiliary gas, 5 arbitrary units; spray voltage, $4.5 \mathrm{kV}$; capillary voltage, $11 \mathrm{~V}$; capillary temperature, $220{ }^{\circ} \mathrm{C}$; tube lens offset, $35 \mathrm{~V}$. Product ion spectra were acquired using an isolation width of $6 \mathrm{~m} / \mathrm{z}$ units ( $1 \mathrm{~m} / \mathrm{z}$ unit for the H/D exchange experiments), an activation Q of 0.250 and an activation time of 30 msec. The normalised collision energy was set to $50 \%$ and WideBand activation was switched on. Acquisitions were performed for twenty scans. Helium was used as the buffer gas. Data acquisition was performed using XCalibur version 1.2 (Thermo Fisher Scientific, San Jose, CA, USA).

Accurate mass measurements were performed using an Apex III FT-ICR mass spectrometer (Bruker, Billerica, MA, USA) equipped with a 4.7 Tesla actively shielded superconducting magnet, an Infinity cylindrical analyser cell and an Apollo ESI source. Positive ion ESI was performed by infusing the solutions directly into the source at a constant flow rate of

$3 \mu \mathrm{L} \mathrm{min}{ }^{-1}$. Nitrogen was used as the drying gas. The source conditions were; capillary voltage, $-4.5 \mathrm{kV}$; end plate voltage, $-3.8 \mathrm{kV}$; capillary exit voltage, $100 \mathrm{~V}$; skimmer $1,11 \mathrm{~V}$; skimmer 2, $6 \mathrm{~V}$; Offset, 1.25; Rf amplitude, $600 \mathrm{~Hz}$; dry gas temperature, $250{ }^{\circ} \mathrm{C}$; dry gas flow rate, 30 arbitrary units; nebulising gas pressure, 50 psi. The excitation amplitude was $3.7 \mathrm{~dB}$. The product ion spectra were acquired for eight scans using $1024 \mathrm{~K}$ data points. Argon was used as the collision gas. The MS/MS parameters were; corr sweep pulse length, 1000 Hsec; corr sweep attenuation, 37.5 dB; ejection safety belt, $3000 \mathrm{~Hz}$; user pulse length, 
$1000 \mu \mathrm{sec}$; ion activation pulse length, $250000 \mu \mathrm{sec}$; ion activation attenuation, $37.2 \mathrm{~dB}$; frequency offset from activation mass, - $500 \mathrm{~Hz}$; user delay length, $3 \mathrm{sec}$. The instrument was calibrated using the first-generation product ion spectrum of protonated reserpine. The

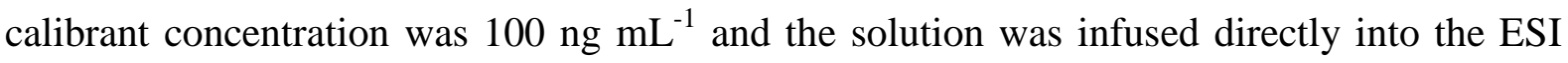
source at a constant flow rate of $3 \mu \mathrm{L} \mathrm{min}{ }^{-1}$. Data acquisition was performed using XMass version 7.0.8 (Bruker, Billerica, MA, USA).

\section{Results and discussion}

During a project to identify product ions and losses that facilitate rapid and definitive identification of pharmaceutical drug metabolites, the dissociation of a model S-oxidised metabolite, 3-dimethylaminomethyl-4-(4-methanesulfinyl-3-methyl-phenoxy)benzenesulfonamide (1) (Figure 1), was investigated. The first generation product ion spectrum of the protonated molecule is shown in Figure 2. It was hypothesised that the ion at $\mathrm{m} / \mathrm{z} 321$ was due to the loss of the methyl sulfoxide group. Thus, the dissociation was thought to be of potential use in metabolite identification; the $62 \mathrm{~m} / \mathrm{z}$ unit loss being assigned as methanethial, S-oxide $\left(\mathrm{CH}_{2} \mathrm{SO}\right)$. S-oxidation of the parent compound produces an ideal candidate for the loss of $62 \mathrm{~m} / \mathrm{z}$ units through the well-documented processes of chargeremote fragmentation ${ }^{12}$ and 1,3-proton shifts ${ }^{13}$ via four-centred rearrangements (Figure 3). This hypothesis was further attractive due to the absence of the same $62 \mathrm{~m} / \mathrm{z}$ unit loss from the parent compound (data not shown). Thus, it was postulated that the loss involved the oxygen atom. The dissociation was thought to be energetically favourable due to the conformance of the postulated product ion with the even-electron rule. ${ }^{14}$ However, the loss could not be confirmed by a change in the ${ }^{34} \mathrm{~S}$ isotope pattern for the product ion compared to the precursor ion due to the difficulties associated with observing the true isotope pattern using a QIT mass spectrometer. The overlapping of the A + 2 peak with the peak due to the lightest isotopes of the product ion at $\mathrm{m} / \mathrm{z} 323$ further precluded this approach to structural elucidation.

It was noted that the $62 \mathrm{~m} / \mathrm{z}$ unit loss involved none of the three exchangeable hydrogen atoms on the precursor ion. Thus, if the assignment of the loss was correct, the $\mathrm{m} / \mathrm{z}$ of the product ion would shift by $3 \mathrm{~m} / \mathrm{z}$ units to $\mathrm{m} / \mathrm{z} 324$ in the product ion spectrum of the fully exchanged, deuterated species (Figure 4). The absence of a peak at $\mathrm{m} / \mathrm{z} 324$ in the deuterated sample indicated that the initially posited loss was incorrect. Further, an additional peak was 
observed at $\mathrm{m} / \mathrm{z} 323$ in the product ion spectrum of the fully, exchanged deuterated species that was not seen for the protonated molecule. It should be noted that this ion was different to that seen at $m / z 323$ in the product ion spectrum of the protonated molecule, which was mass shifted to $\mathrm{m} / \mathrm{z} 325$ under deuterated conditions. The observation of an additional peak led to the deduction that two nominally isobaric product ions were formed through dissociation of the protonated species. The product ions only became mass resolved when the fully exchanged, deuterated molecule was fragmented due to the retention of different numbers of deuterium atoms by the two product ions. Thus, the H/D exchange experiment showed that two losses of $62 \mathrm{~m} / \mathrm{z}$ units took place, neither of which was that initially postulated. The loss of all of the deuterium atoms to form the product ion at $\mathrm{m} / \mathrm{z} 321$ indicated that the primary amine group had been lost during the dissociation. This was because exchange of two hydrogen atoms for deuterium labels would have occurred at this functional group. The peak at $\mathrm{m} / \mathrm{z} 323$, formed through a loss involving one deuterium atom, indicated that this product ion retained the primary amine. Further, it suggested that the ionising deuteron was lost during the dissociation, and hence the loss included a site of ionisation.

Further experimentation was undertaken to characterise the two losses of $62 \mathrm{~m} / \mathrm{z}$ units. To this end, the first generation product ion spectrum of a deuterium labelled analogue, 3-dimethyl${ }^{2} \mathrm{H}_{6}$-aminomethyl-4-(4-methanesulfinyl-3-methyl-phenoxy)-benzenesulfonamide (2) (Figure 5), was acquired (Figure 6). In the mass range of interest, the product ion spectrum resembled that of protonated 1 with only two peaks being observed. This indicated that the product ions were nominally isobaric again and neither product ion retained the six deuterium labels. Thus, both losses of $62 \mathrm{~m} / \mathrm{z}$ units involved the tertiary amine group. The H/D exchange experiment had shown that one product ion also lost the primary amine group; thus the overall loss was postulated to be $\mathrm{C}_{2} \mathrm{H}_{10} \mathrm{~N}_{2}$. The same experiment had also shown that one product ion retained the primary amine and lost the ionising deuteron. From this information, it was deduced that the tertiary amine group was a site of protonation. Further, it suggested that the remaining loss of $17 \mathrm{~m} / \mathrm{z}$ units for the product ion that retained the primary amine group (62 $\mathrm{m} / \mathrm{z}$ units minus $45 \mathrm{~m} / \mathrm{z}$ units for the tertiary amine group plus the ionising proton) was likely to be a hydroxyl radical; an overall proposed loss of $\mathrm{C}_{2} \mathrm{H}_{8} \mathrm{NO}^{\circ}$.

To confirm the hypothesised losses, accurate mass measurements were performed using Fourier transform-ion cyclotron resonance (FT-ICR) mass spectrometry. Figure 7 shows the first generation product ion spectrum of protonated $\mathbf{1}$ in the mass range of interest. The high 
resolving power of the FT-ICR mass spectrometer allowed mass resolution of the two nominally isobaric ions at $\mathrm{m} / \mathrm{z} 321$. The lowest error elemental formulae, given the precursor ion formula, were consistent with those postulated and confirmed that the two losses of $62 \mathrm{~m} / \mathrm{z}$ units were $\mathrm{C}_{2} \mathrm{H}_{10} \mathrm{~N}_{2}$ and $\mathrm{C}_{2} \mathrm{H}_{8} \mathrm{NO}^{\circ}$.

The verification of the elemental composition of the losses allowed postulation of dissociation mechanisms leading to the decomposition of the precursor ion. The ion with an elemental formula of $\mathrm{C}_{15} \mathrm{H}_{13} \mathrm{O}_{4} \mathrm{~S}_{2}{ }^{+}$(3) had a ring plus double bond equivalents value of 9.5, confirming that it was an even-electron ion. ${ }^{15}$ Because of the deficiency of this value by 0.5 due to the addition of a proton to the molecule ${ }^{16}$, the number of rings plus double bond equivalents in the product ion must have been 10. Figure 8 shows a proposed mechanism leading to the formation of a product ion which satisfies this value; the double bonds of the sulfone group are discounted due to sulfur not being in its lowest valence state. ${ }^{15}$ Protonation is postulated on the primary amine to facilitate the loss of the two exchangeable hydrogen atoms as part of a molecule of ammonia. The posited product ion structure is predominately planar as evinced by the molecular model in Figure 9. This is likely to provide the driving force for the energetic rearrangement involving a high energy proton shift leading to the loss of dimethylmine. ${ }^{17}$ The planar structure will allow easy delocalisation of the charge, making the formation of the product ion thermodynamically favourable.

The ion with an elemental formula of $\mathrm{C}_{15} \mathrm{H}_{15} \mathrm{NO}_{3} \mathrm{~S}_{2}{ }^{+\bullet}$ (4) had a ring plus double bond equivalents value of 10 i.e. an odd-electron species. ${ }^{15}$ Figure 10 shows a proposed mechanism to form a radical product ion structure that is consistent with this value. Again, the posited product ion structure is predominately planar (Figure 11), thus allowing facile delocalisation of the charge, and also the radical site. This is particularly important due to the energetically unfavourable formation of a radical product ion from an even electron precursor. ${ }^{18}$ However, the resonance stabilisation across the two aromatic rings, and the sulfone and sulfoxide functionalities, is likely to facilitate this dissociation.

Both proposed dissociation mechanisms involve the loss of dimethylamine. However, the pathways leading to this loss differ for the two product ions. The formation of $\mathbf{3}$ is postulated to involve the loss of dimethylamine facilitated by the shift of a non-exchangeable hydrogen atom. On the other hand, the formation of $\mathbf{4}$ proceeds via protonation of the tertiary amine followed by a charge-directed inductive cleavage of the carbon-nitrogen bond. Thus, an 
exchangeable hydrogen atom i.e. the ionising proton (deuteron), is proposed to facilitate the loss of dimethylamine in this instance. The loss of dimethylamine was also observed in the first generation product ion spectrum of protonated 1 (Figure 2; product ion at $\mathrm{m} / \mathrm{z} 338$ ). The dissociation of the fully exchanged, deuterated species (Figure 4) showed that the loss increased by $1 \mathrm{~m} / \mathrm{z}$ unit to produce a peak at $\mathrm{m} / \mathrm{z} 340$, demonstrating the involvement of a single exchangeable hydrogen atom in the fragmentation. The protonated molecule can not be spatial arranged such that the two exchangeable hydrogen atoms on the primary amine can be in close proximity to the tertiary amine functionality to allow intramolecular transfer. Thus, they can be excluded as being the source of the exchangeable hydrogen atom involved in the loss of dimethylamine. Hence, the exchangeable hydrogen atom must be the ionising proton (deuteron). From the two mechanisms postulated for the losses of $62 \mathrm{~m} / \mathrm{z}$ units (Figures 8 and 10), only one involves protonation at the tertiary amine and the loss of dimethylamine facilitated by an exchangeable hydrogen atom i.e. the loss of $\mathrm{C}_{2} \mathrm{H}_{8} \mathrm{NO}$. Protonation at the primary amine is inferred for the loss of $\mathrm{C}_{2} \mathrm{H}_{10} \mathrm{~N}_{2}$ to facilitate expulsion of a molecule of ammonia from the precursor ion. This could not be confirmed using H/D exchange experiments because the loss of ammonia is not observed in the first generation product ion spectrum of protonated 1 (Figure 2). However, supportive evidence for the proposed mechanisms was obtained through acquisition of the second generation product ion spectrum of fully exchanged, deuterated 1 using the ion at $\mathrm{m} / \mathrm{z} 340$ as the precursor ion for the second stage of mass analysis (Figure 12). The dissociation of the fully exchanged, deuterated molecule, rather than the protonated molecule, was investigated so that the product ions would be mass resolved on the QIT mass spectrometer if they were both formed. In the mass range of interest, a product ion was observed at $\mathrm{m} / \mathrm{z} 323$, which suggested that $\mathbf{4}$ is formed through protonation at the tertiary amine, followed by the loss of dimethylamine and then expulsion of a hydroxyl radical. The mass difference of $17 \mathrm{~m} / \mathrm{z}$ units between the precursor ion for the second stage of mass analysis and the final product ion indicated that the loss of the hydroxyl radical involved a non-exchangeable hydrogen atom. If an exchangeable hydrogen atom was involved, under deuterated conditions the loss would become a deuterohydroxyl radical and the mass loss would increase to $18 \mathrm{~m} / \mathrm{z}$ units. The observations were consistent with the proposed mechanism for the dissociation (Figure 10). No peak was observed at $m / z$ 321, which indicated that a second dissociation pathway exists for the loss of dimethylamine. This provided supportive evidence for a loss of dimethylamine facilitated by the shift of a non-exchangeable hydrogen atom, which is consistent with the proposed mechanism (Figure 8). The absence of the loss of ammonia and dimethylamine facilitated by 
a non-exchangeable hydrogen atom in the first generation product ion spectrum of protonated 1 suggested that the dissociation was concerted, or at least the intermediates underwent rapid dissociation to the final product ion. Further, the H/D exchange experiments suggested that $\mathbf{1}$ had more than one site of protonation in the gas-phase because the loss of the tertiary amine group was not via protonation at that site in the formation of $\mathbf{3}$. Thus, it was plausible that the molecule could protonate at the primary amine, further supporting the mechanism proposal.

The discussed dissociation has implications for structural elucidation using CID product ion spectra, particularly pharmaceutical drug metabolite identification studies. The structure of a metabolite must be deduced from its product ion spectrum. From the low resolution product ion spectrum of protonated $\mathbf{1}, S$-oxidation is likely to be proposed based upon the initial assignment of the $62 \mathrm{~m} / \mathrm{z}$ units loss as methanethial, $S$-oxide via a four-centred rearrangement. In this case, the proposal of S-oxidation would have been correct, but the assignments of ions leading to this proposal would have been incorrect. If this were an unknown metabolite, oxidation of the parent compound could have occurred, for example, through aromatic hydroxylation of the 1-methyl-2-methylsulfanyl-benzene ring and potentially not affected either of the actual losses of $62 \mathrm{~m} / \mathrm{z}$ units that took place. The attractive nature of $S$-oxidation facilitating the loss of methanethial, $S$-oxide via a fourcentred rearrangement may lead to mis-assignment of the ions in the CID spectrum. Hence time and money may be spent synthesising analogues and/or homologues to protect the methyl sulfide functionality of the parent compound only to discover that the hypothesised site of oxidation is incorrect. The discussed example highlights the possibility of unexpected rearrangements under CID conditions, which could easily lead to incorrect assignment of ion structure, and hence sites of metabolism in drug discovery studies. A simple and cost effective approach to reduce the likelihood of this occurrence is the use of H/D exchange experiments, which provide a straightforward method of introducing isotopic labels into the compound. Thus, dissociations proposed to involve exchangeable hydrogen atoms can be verified. Further, employment of mass spectrometers capable of high resolution, even when coupled to fast chromatography as utilised in metabolite identification studies, would also reduce the possibility of incorrect ion structure assignment. The study has also been of relevance to the understanding of fragmentation under CID conditions. The actual dissociations involved more complex rearrangements than that initially proposed, and also the energetically unfavourable formation of a radical product ion. However, the final shape of the product ion appears to have a bearing on the dissociation pathway followed. The initially 
proposed product ion structure formed through the loss of methanethial, S-oxide has free rotation about the ether linkage. The product ions actually formed are posited to be predominately planar, thus allowing easier delocalisation of the charge. This may suggest that the energetic favourability of the product ion structure can outweigh the facileness of the rearrangement and provide the driving force for dissociation; suggesting less dependence on the kinetics of the fragmentation under CID conditions compared to electron ionisation (EI) conditions. ${ }^{1}$ Furthermore, the study indicated that H/D exchange experiments can be useful in determining sites of gas-phase protonation under ESI-CID conditions, which has been demonstrated in a previous study ${ }^{19}$, thus allowing rationalisation of dissociation mechanisms.

\section{Conclusions}

The experimental investigation of the dissociation of a model $S$-oxidised metabolite has been presented. The ion at $\mathrm{m} / \mathrm{z} 321$ observed in the low resolution CID product ion spectrum was initially assigned as being formed through the loss of methanethial, $S$-oxide from the protonated molecule. It was thought that this loss could be used to assign the site of metabolism as the sulfur of the methyl sulfide group. Subsequent deuterium labelling experiments and accurate mass measurements acquired using a high resolution CID product ion spectrum proved that the initial assignment was incorrect. Instead, two unexpected rearrangements lead to the formation of nominally isobaric ions. Only through the use of H/D exchange experiments and high resolution mass spectrometry was it possible to mass resolve these species. Mechanisms consistent with the experimental findings were postulated and supportive evidence for the proposals was obtained through the acquisition of a second generation product ion spectrum. The proposed planar product ion structures were concluded to provide the driving force for the rearrangements, particularly in the formation of an energetically unfavourable radical cation. The study was of relevance to pharmaceutical drug metabolite identification because it demonstrated that a rigorous analytical approach is required to ensure that incorrect assignment of the sites of metabolism of novel chemical entities is precluded. A greater understanding of fragmentation under CID conditions was also obtained, with the shape of the product ion apparently offsetting the complexity of the rearrangement required to form it. Further, the data verified the usefulness of H/D exchange experiments in determining sites of protonation under ESI-CID conditions. 


\section{Acknowledgements}

This work was financially supported by the UK Biotechnology and Biological Research Council (BBSRC grant number BBS/S/N/2006/13093) and Pfizer Global Research and Development. The authors wish to thank Dr. Katerina Klagkou for helpful discussions relating to the dissociation mechanisms. 


\section{Figures}

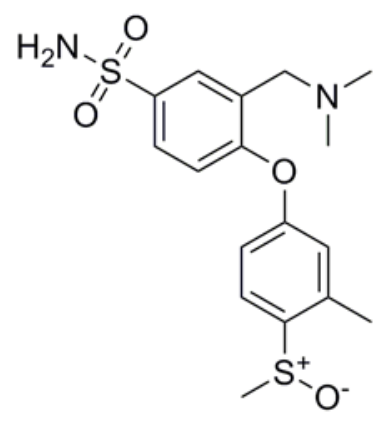

Figure 1. Molecular structure of 3-dimethylaminomethyl-4-(4-methanesulfinyl-3-methyl-phenoxy)-benzenesulfonamide (1) 


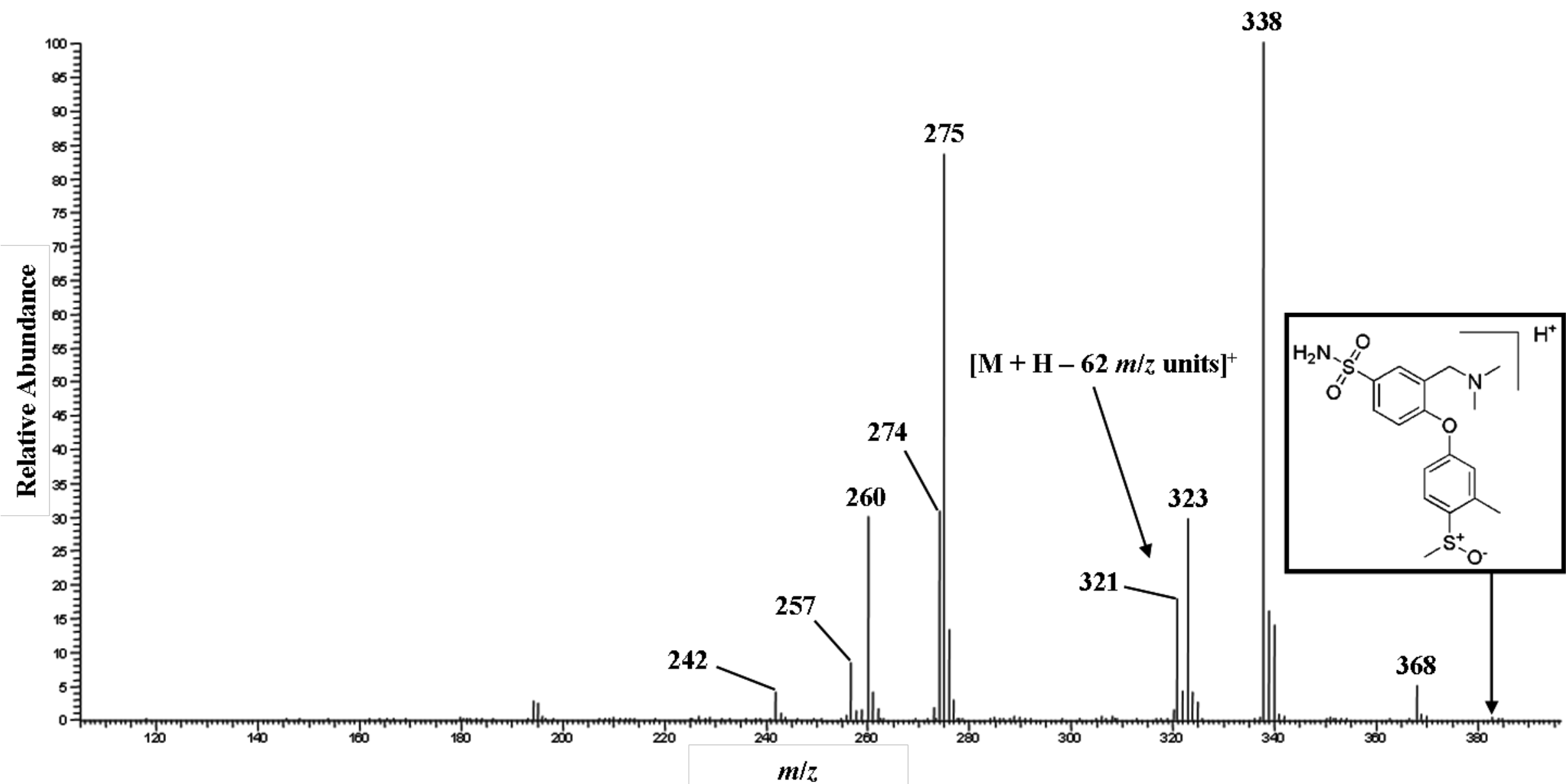

Figure 2. First generation product ion spectrum of protonated $\mathbf{1}$ acquired using a LCQ Classic QIT mass spectrometer with WideBand activation on 

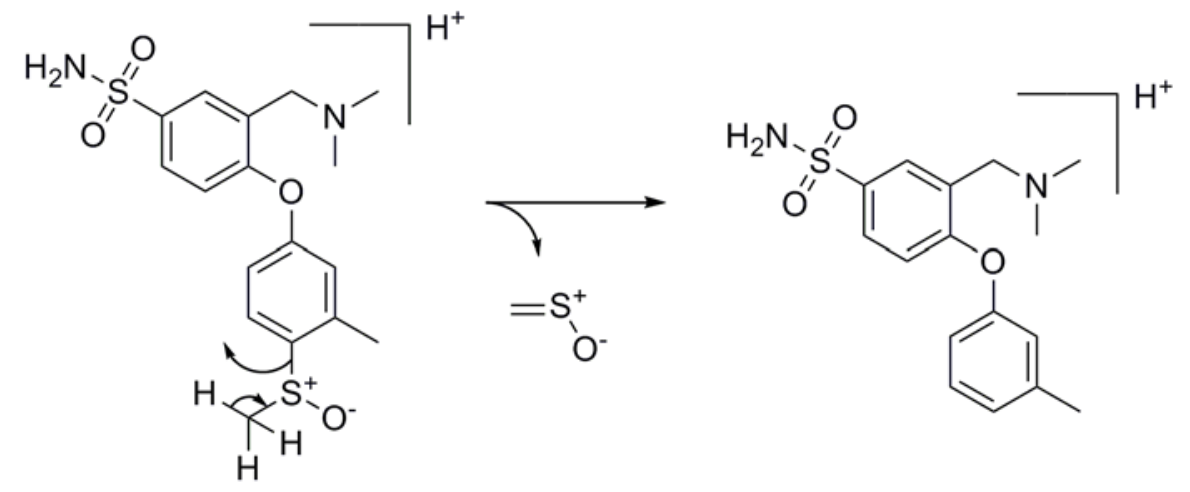

Figure 3. Proposed mechanism for the loss of methanethial, S-oxide from protonated $\mathbf{1}$ via a four-centred rearrangement 


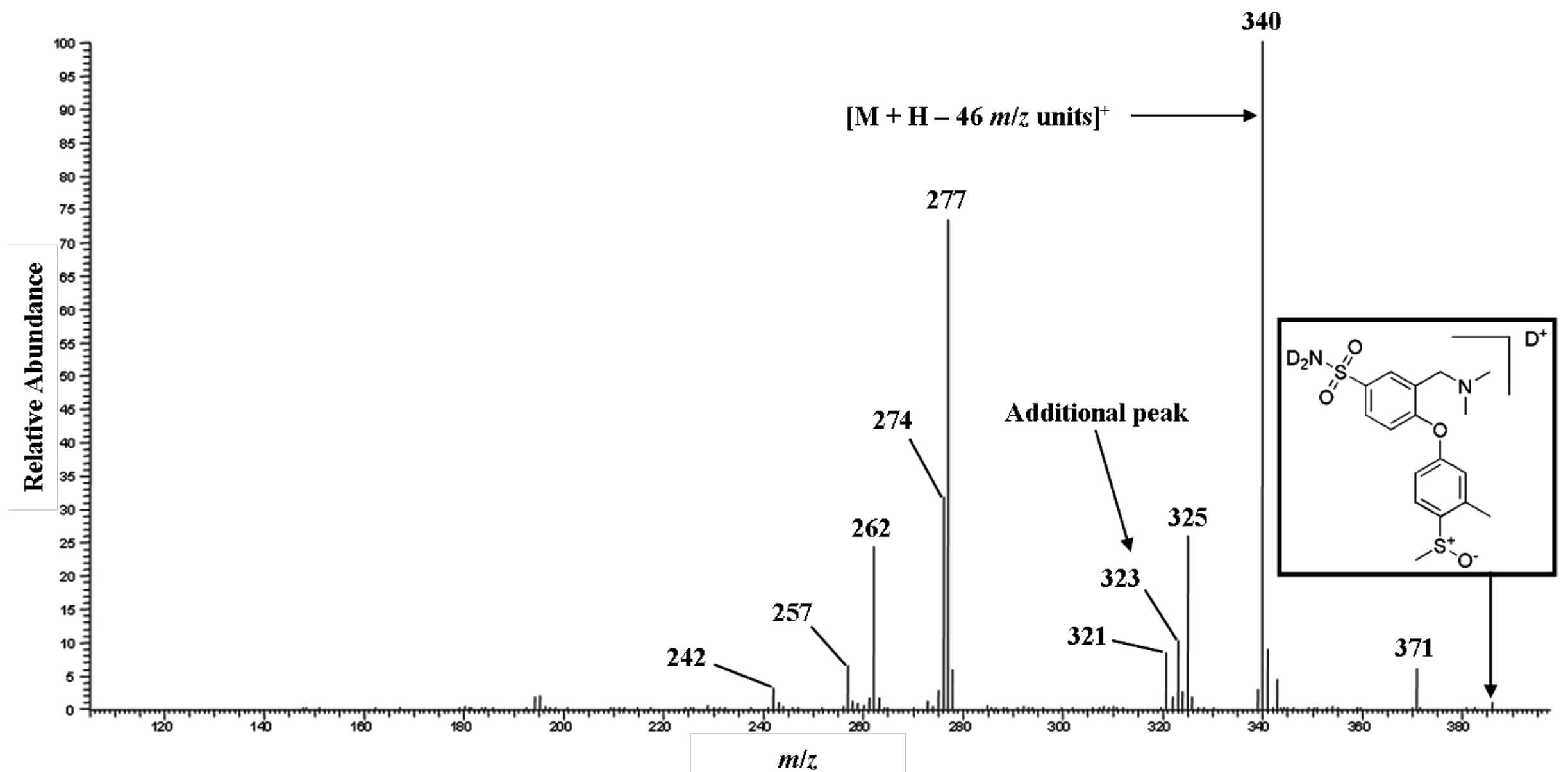

Figure 4. First generation product ion spectrum of fully exchanged, deuterated 1 acquired using a LCQ Classic QIT mass spectrometer with WideBand activation on 


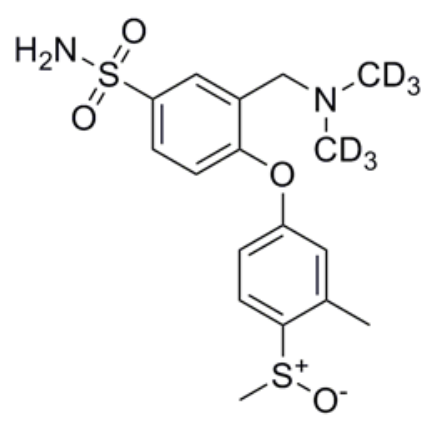

Figure 5. Molecular structure of 3-dimethyl- ${ }^{2} \mathrm{H}_{6}$-aminomethyl-4-(4-methanesulfinyl-3-methyl-phenoxy)-benzenesulfonamide (2) 


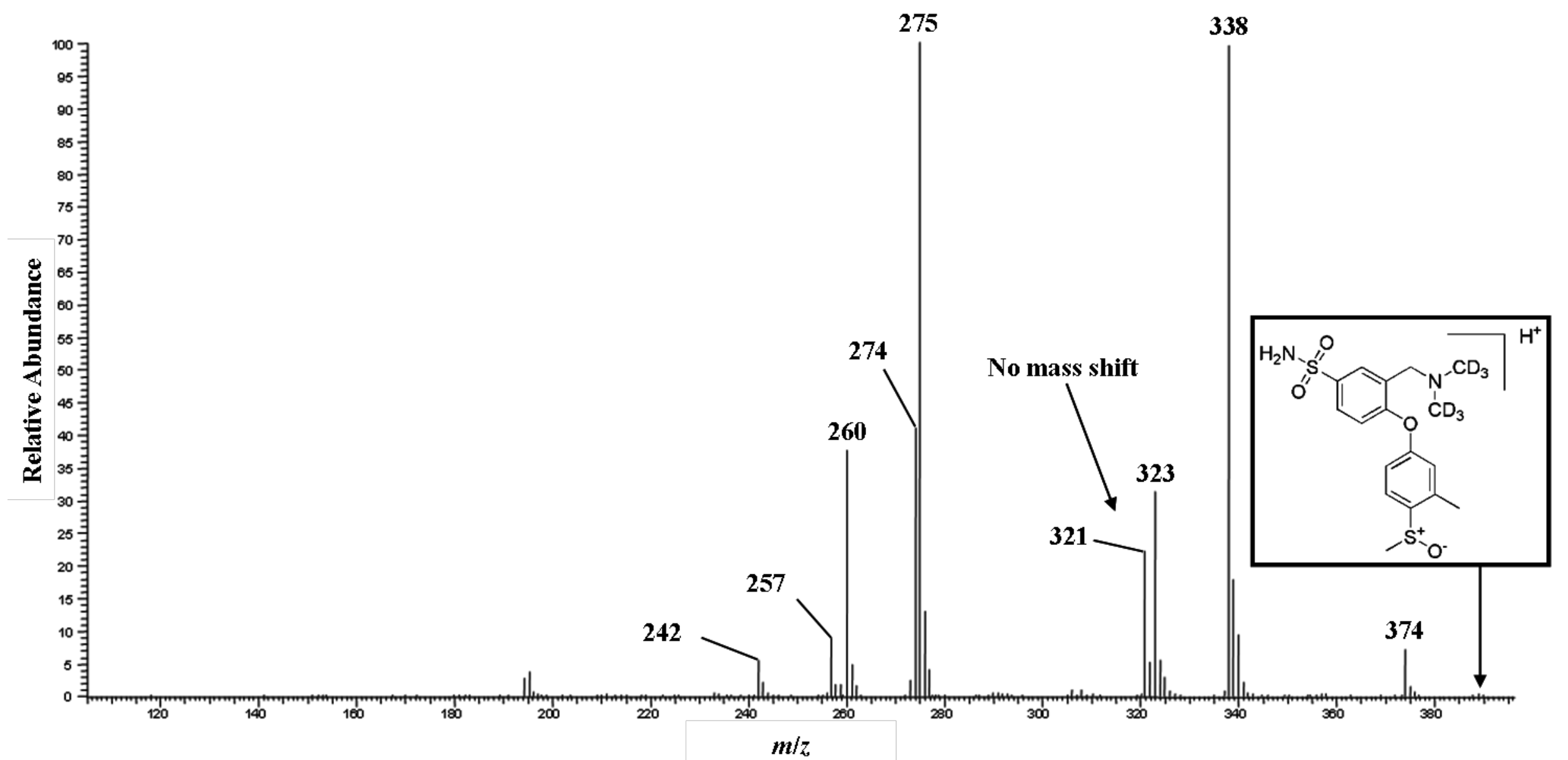

Figure 6. First generation product ion spectrum of protonated 2 acquired using a LCQ Classic QIT mass spectrometer with WideBand activation on 


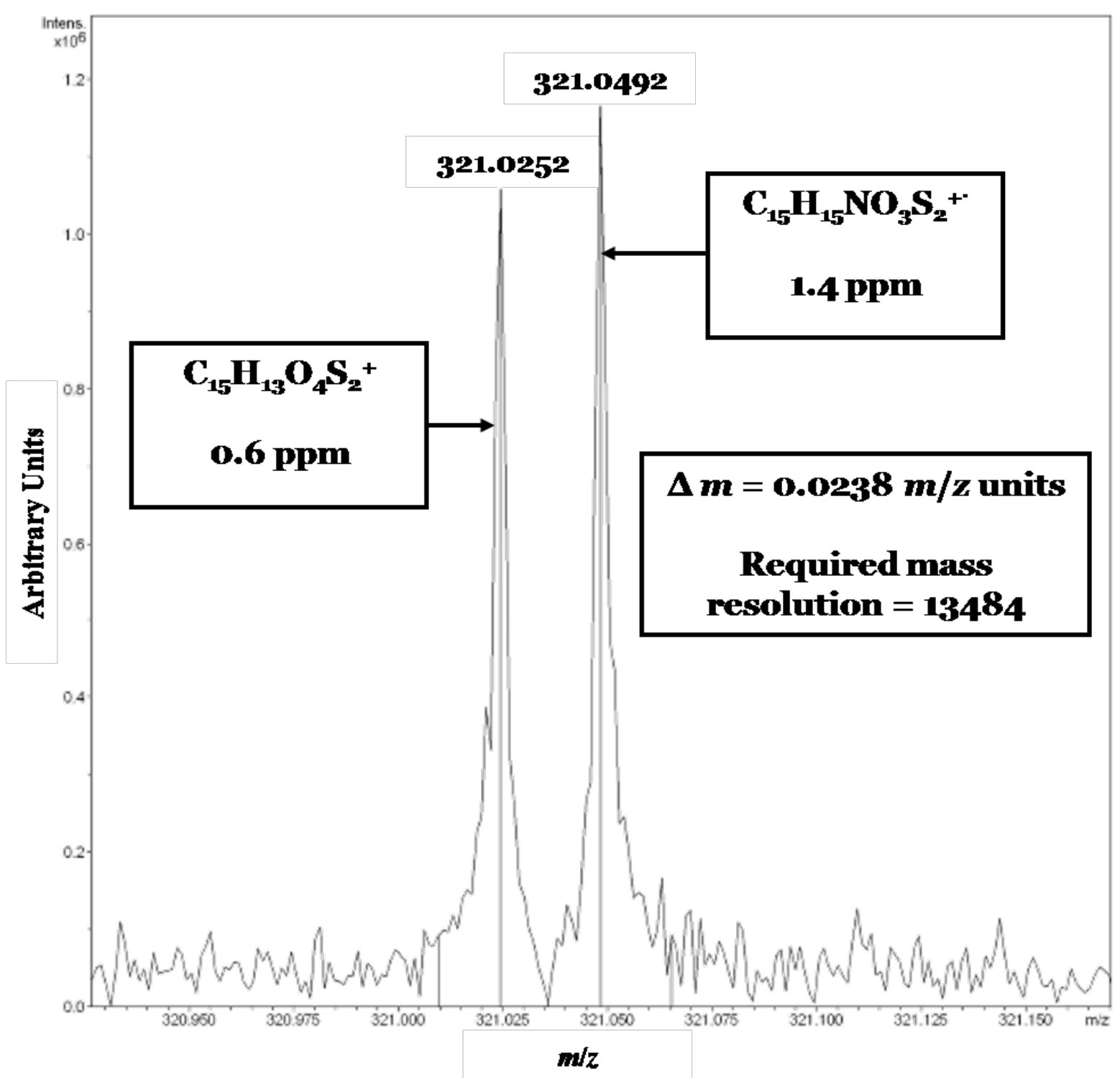

Figure 7. First generation product ion spectrum of protonated $\mathbf{1}$ acquired using an Apex III FT-ICR mass spectrometer 

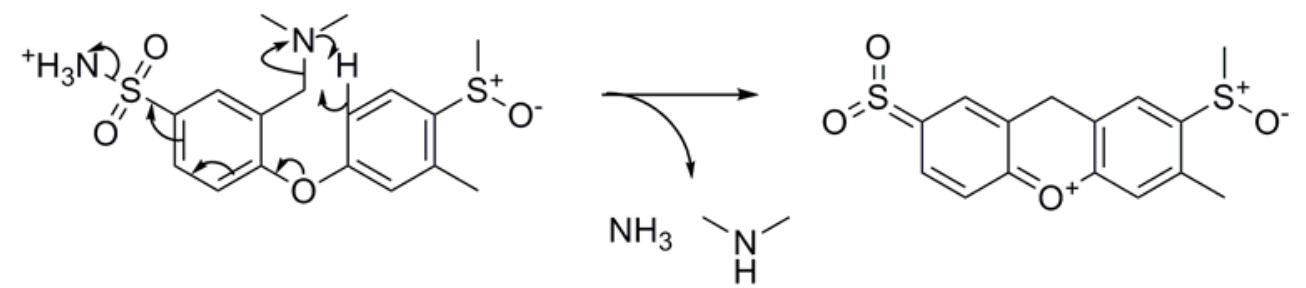

Figure 8. Proposed mechanism for the formation of 3 through the loss of $\mathrm{C}_{2} \mathrm{H}_{10} \mathrm{~N}_{2}$ as molecules of ammonia and dimethylamine from protonated 1 

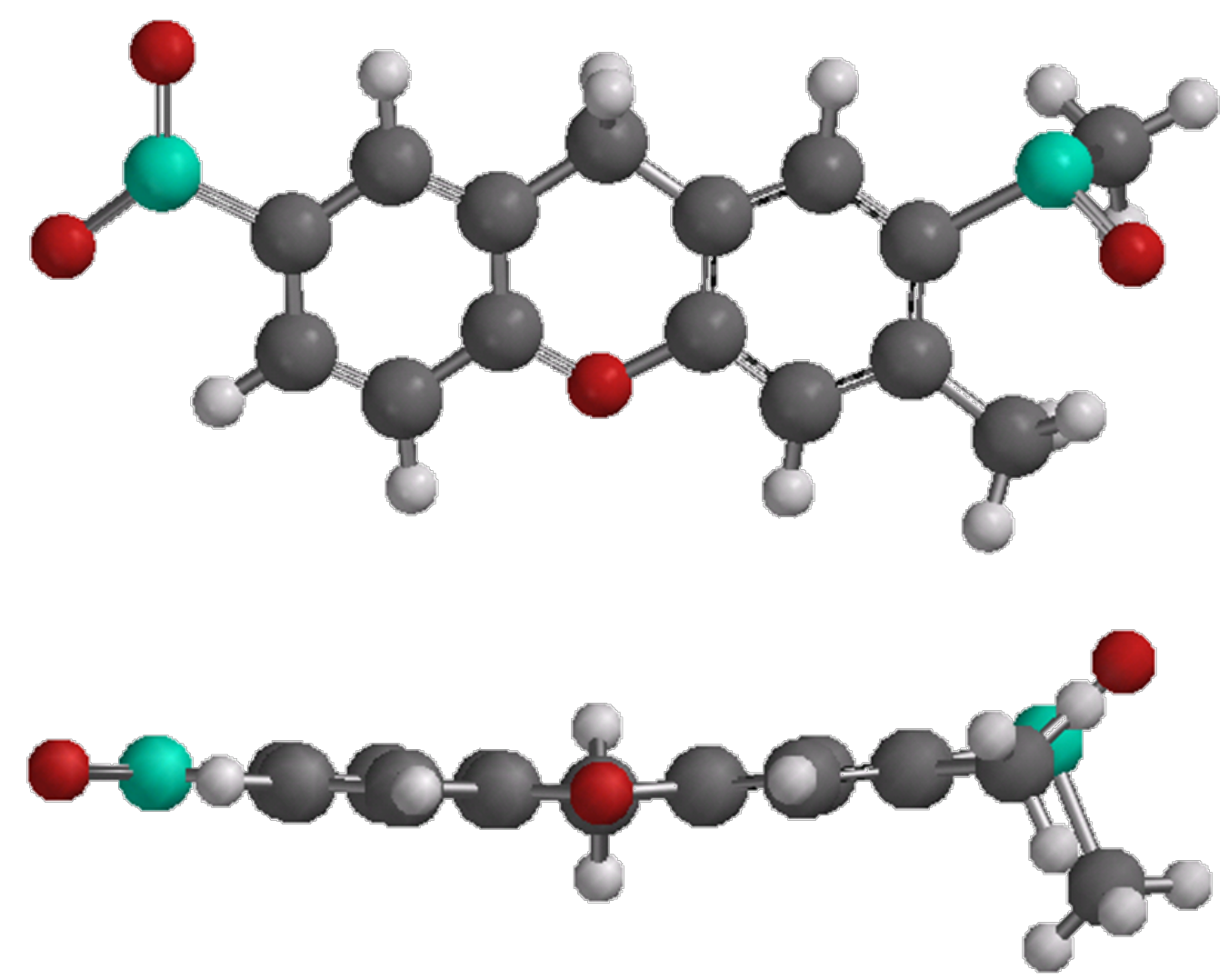

Figure 9. Molecular models of $\mathbf{3}$ demonstrating the predominately planar structure of the product ion 


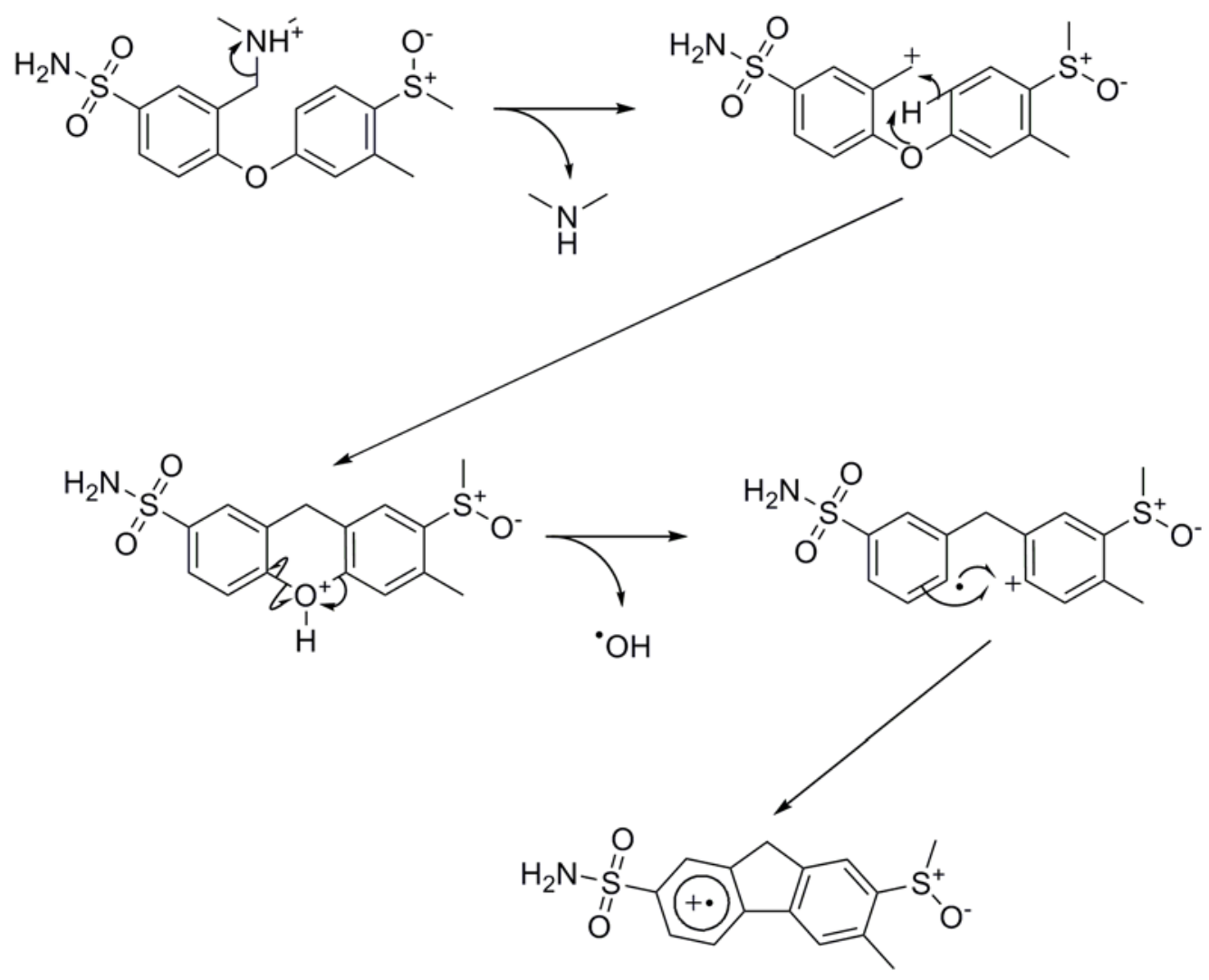

Figure 10. Proposed mechanism for the formation of 4 through the loss of $\mathrm{C}_{2} \mathrm{H}_{8} \mathrm{NO}{ }^{\bullet}$ as a molecule dimethylamine and a hydroxyl radical from protonated 1 

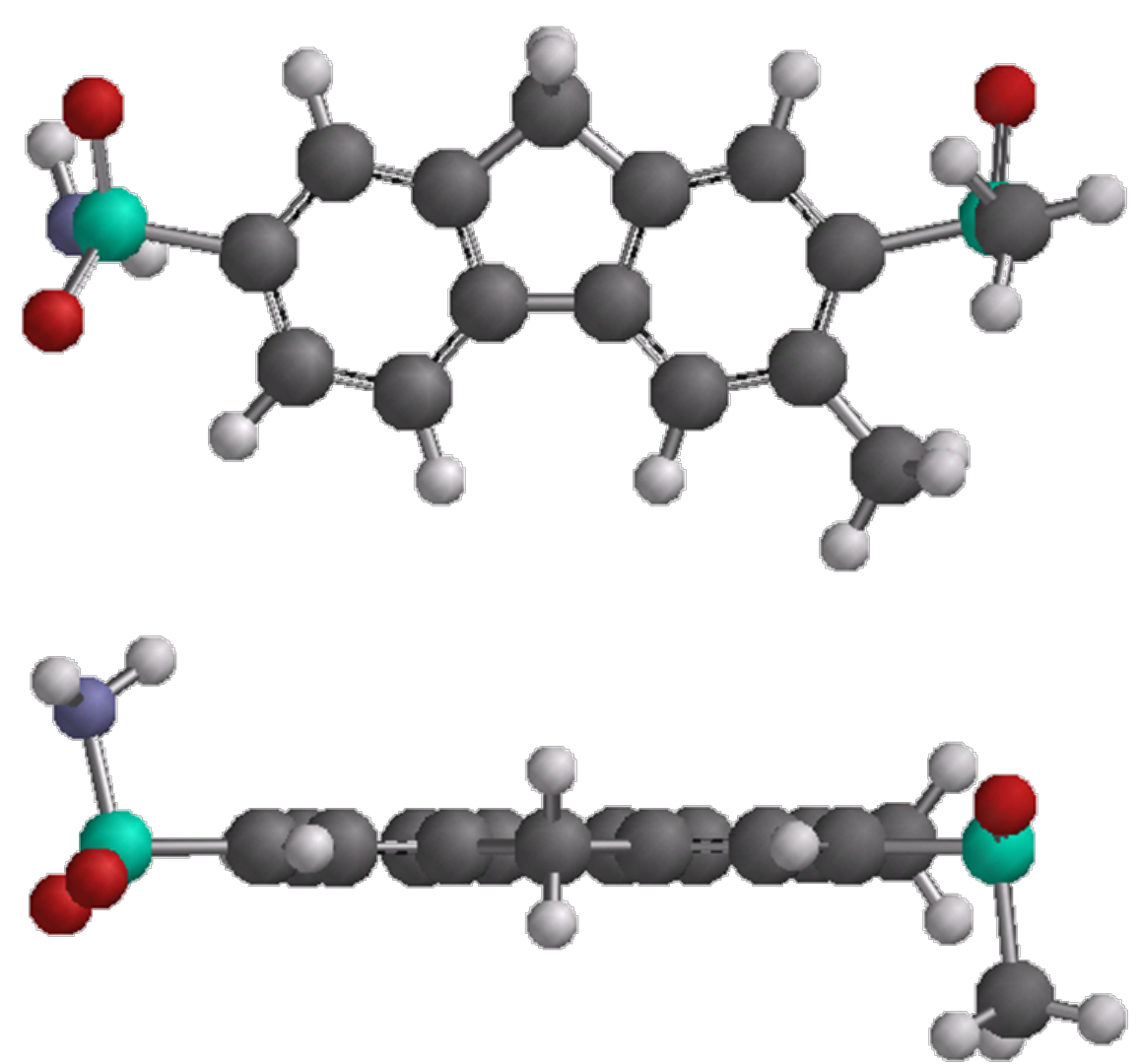

Figure 11. Molecular models of $\mathbf{4}$ demonstrating the predominately planar structure of the product ion 


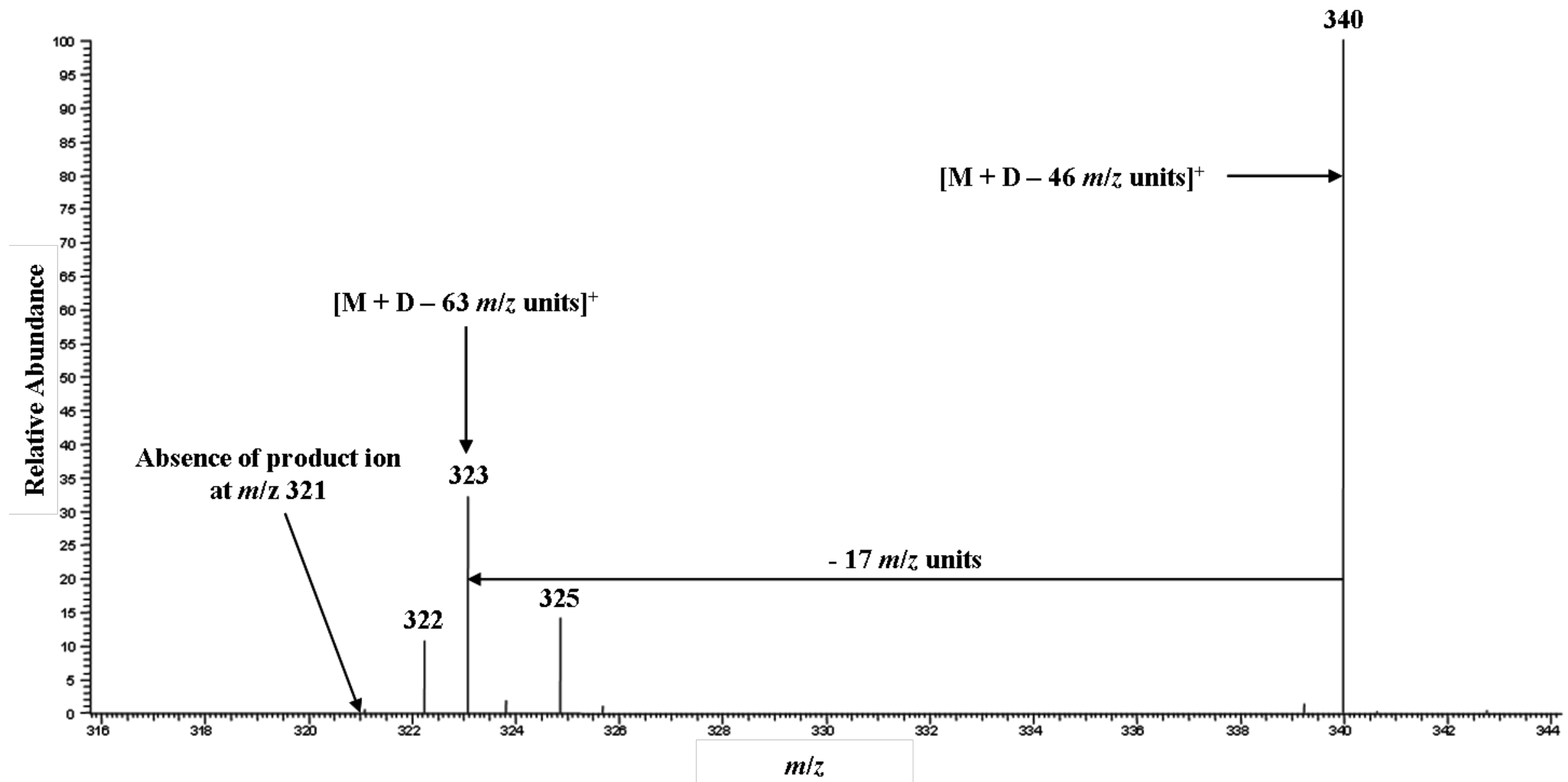

Figure 12. Second generation product ion spectrum of fully exchanged, deuterated $\mathbf{1}$ using the ion at $\mathrm{m} / \mathrm{z} 340$ as the precursor ion for the second stage of mass analysis acquired using a LCQ Classic QIT mass spectrometer with WideBand activation on 


\section{References}

1. C. Prakash, C. L. Shaffer and A. Nedderman, Mass Spectrom. Rev., 2007, 26, 340369.

2. R. Ramanathan, D. L. McKenzie, M. Tugnait and K. Siebenaler, J. Pharm. Biomed. Anal., 2002, 28, 945-951.

3. A. Fura, Drug Discov. Today, 2006, 11, 133-142.

4. S.-W. Myung, H.-Y. Kim, H.-K. Min, D.-H. Kim, M. Kim, H.-W. Choo, H. S. Lee, J.-K. Kim and C. I. Hong, Rapid Commun. Mass Spectrom., 2002, 16, 2048-2053.

5. $\quad$ S. Ma and R. Subramanian, J. Mass Spectrom., 2006, 41, 1121-1139.

6. $\quad$ K. Biemann, J. Am. Soc. Mass Spectrom., 2002, 13, 1254-1272.

7. P. Wright, A. Alex, D. Gibson, R. Jones and P. Macrae, Rapid Commun. Mass Spectrom., 2005, 19, 2005-2014.

8. P. R. Tiller, C. Raab and C. E. C. A. Hop, J. Mass Spectrom., 2001, 36, 344-345.

9. A. Cartoni, M. Altamura, F. Animati, G. Balacco, R. Cosi, A. Ettorre, A. Madami and A. Triolo, J. Mass Spectrom., 2002, 37, 1258-1265.

10. R. V. Vachet, B. M. Bishop, B. W. Erickson and G. L. Glish, J. Am. Chem. Soc., 1997, 119, 5481-5488.

11. A. G. Craig and S. W. Taylor, J. Am. Soc. Mass Spectrom., 2001, 12, 470-474.

12. C. Cheng and M. L. Gross, Mass Spectrom. Rev., 2000, 19, 398-420.

13. C. E. Hudson and D. J. McAdoo, J. Am. Soc. Mass Spectrom., 2004, 15, 972-981.

14. M. Karni and A. Mandelbaum, Org. Mass Spectrom., 1980, 15, 53-64.

15. F. W. McLafferty and F. Turecek, Interpretation of Mass Spectra, 4th Edn., University Science Books, Sausalito, 1993.

16. V. Pellegrin, J. Chem. Educ., 1983, 60, 626-633.

17. J. T. Bursey, M. M. Bursey and D. G. I. Kingston, Chem. Rev., 1973, 73, 191-234.

18. R. G. Cooks, Org. Mass Spectrom., 1969, 2, 481-519.

19. S. W. Holman, P. Wright and G. J. Langley, Rapid Commun. Mass Spectrom., 2008, 22, 2355-2365. 
High-throughput approaches towards the definitive identification of pharmaceutical drug metabolites. 2 . An example of how unexpected dissociation behaviour could preclude correct assignment of sites of metabolism

\author{
Stephen W. Holman ${ }^{1}$, Patricia Wright ${ }^{2}$ and G. John Langley ${ }^{1 *}$ \\ ${ }^{1}$ School of Chemistry, University of Southampton, Southampton, SO17 1BJ, UK \\ ${ }^{2}$ Department of Pharmacokinetics, Dynamics and Metabolism, Pfizer Global Research and \\ Development, Ramsgate Road, Sandwich, CT13 9NJ, UK
}

Correspondence to: G. J. Langley, School of Chemistry, University of Southampton, Southampton, SO17 1BJ, UK,

E-mail: g.j.langley@soton.ac.uk

Tel: +44 2380593320

Fax: +44 2380597641 


\begin{abstract}
S-oxidation is a common metabolic route for sulphur containing compounds. When studying the fragmentation of some chemically synthesised sulphoxides, two unexpected losses of $62 \mathrm{~m} / \mathrm{z}$ units were observed in the collision-induced dissociation (CID) product ion spectrum protonated 3-dimethylaminomethyl-4-(4-methanesulfinyl-3-methyl-phenoxy)benzenesulfonamide. A single loss was initially assigned using the low resolution product ion spectrum, acquired by electrospray ionisation-quadrupole ion trap-mass spectrometry (ESIQIT-MS), as methanethial, S-oxide with the dissociation proceeding via a charge-remote, four-centred rearrangement. This assignment was consistent with well-documented hydrogen rearrangements in the literature. Further, the loss was not observed for the parent compound. Thus, it was inferred that the site of metabolism was involved in the dissociation and the attractive nature of the four-centred rearrangement meant that the loss of methanethial, $S$ oxide was a logical assignment. However, deuterium labelling experiments and accurate mass measurements, performed using electrospray ionisation-Fourier Transform Ion Cyclotron Resonance-mass spectrometry (ESI-FTICR-MS), showed that two distinct losses of $62 \mathrm{~m} / \mathrm{z}$ units occur, neither of which was of that initially hypothesised from the low resolution product ion spectrum of the protonated molecule. Mechanisms consistent with the experimental findings are postulated. An $\mathrm{MS}^{3}$ spectrum of the fully exchanged, deuterated species supported the proposed mechanisms by suggesting that 3-dimethylaminomethyl-4-(4methanesulfinyl-3-methyl-phenoxy)-benzenesulfonamide has multiple sites of protonation in the gas phase. The planar structures of the posited product ions are likely to provide the driving force for the rearrangements. The relevance of the observations with regards to pharmaceutical drug metabolite identification are discussed.
\end{abstract}

Keywords: Collision-induced dissociation; Fragmentation; Drug metabolite identification; Drug discovery; H/D exchange.

\title{
Introduction
}

Identification of the metabolites of pharmaceutical compounds is a key stage of drug discovery. Sites on the novel chemical entity prone to biotransformation are identified, thus allowing the pharmacokinetic behaviour of the molecule to be improved through alteration of the compound's structure. ${ }^{1,2}$ Active metabolites, i.e. those possessing pharmacological activity similar, synergistic or inhibitory to that of the parent compound, can be identified to rationalise pharmacodynamic results. ${ }^{3,4}$ Additionally, novel chemical entities that form 
reactive metabolites, resulting in toxicity, can be identified and either modified or eliminated to prevent failure of the drug candidate in the development stage. ${ }^{5}$

During early drug discovery metabolite identification studies, limited amounts of chemical bulk and the need for liquid chromatographic separation may prohibit the routine acquisition of nuclear magnetic resonance (NMR) spectra for definitive structural elucidation. Hence, mass spectrometry is routinely used to assign the sites of metabolism of pharmaceutical drug compounds. The principal method involves comparing the collision-induced dissociation (CID) product ion spectrum of the parent compound with that of the metabolite and looking for mass shifts of the major peaks in these spectra to determine which part of the molecule has been altered i.e. the Shift technique. ${ }^{6}$ However, unambiguous assignments by this approach are frequently not possible, particularly for oxidised metabolites where the biotransformation can take place at carbon, nitrogen or sulfur atoms; all common constituents of pharmaceutical compounds. ${ }^{7}$ Furthermore, the high-throughput nature of modern drug discovery means that multiple mass spectrometric-related structural elucidation experiments e.g. deuterium labelling, derivatisation etc., are rarely performed. Thus, there is a risk that incorrect assignment of sites of metabolism could arise. This is a particular likelihood given that the CID processes for molecules of this type are still not fully understood, with unexpected rearrangements under CID conditions being previously reported in the literature for both small and peptidic molecules. ${ }^{8-11}$ Herein, a further example of unanticipated dissociation behaviour, observed for the model sulfoxide detailed here, demonstrates how incorrect assignment of the sites of metabolism of a novel chemical entity could occur if a rigorous analytical approach is not applied.

\section{Experimental}

\section{Chemicals}

3-dimethylaminomethyl-4-(4-methanesulfinyl-3-methyl-phenoxy)-benzenesulfonamide

and 3-dimethyl- ${ }^{2} \mathrm{H}_{6}$-aminomethyl-4-(4-methanesulfinyl-3-methyl-phenoxy)benzenesulfonamide (2) were synthesised by Pfizer Global Research and Development (Sandwich, UK). Reserpine was purchased from Sigma-Aldrich Company Limited (Gillingham, Dorset, UK). Solutions were prepared in LC-MS grade methanol and analytical 
grade methanoic acid [99.9:0.1, v/v] (Fisher Scientific UK Limited, Loughborough, UK). Solutions of $\mathbf{1}$ and 2 were prepared at $10 \mu \mathrm{g} \mathrm{m}^{-1}$ for the quadrupole ion trap mass spectrometry (QIT-MS) experiments. Further solutions of 1 were prepared at $1 \mu \mathrm{g} \mathrm{mL}{ }^{-1}$ for the Fourier transform ion cyclotron resonance-mass spectrometry (FT-ICR-MS) experiments and $10 \mu \mathrm{g} \mathrm{mL}^{-1}$ in $>99.5 \%$ deuterated methanol and $99.5 \%$ deuterated ethanoic acid [99:1, v/v] (Apollo Scientific Limited, Stockport, UK) for hydrogen/deuterium (H/D) exchange experiments. All chemicals were used without further purification.

\section{Instrumental}

Product ion spectra of either the protonated or fully, exchanged deuterated molecule for each compound were acquired using a LCQ Classic QIT mass spectrometer (Thermo Fisher Scientific, San Jose, CA, USA). Positive ion electrospray (ESI) was performed by infusing the solutions directly into the source at a constant flow rate of $3 \mu \mathrm{L} \mathrm{min}^{-1}$. Nitrogen was used as the sheath and auxiliary gas. The source conditions were; sheath gas, 35 arbitrary units; auxiliary gas, 5 arbitrary units; spray voltage, $4.5 \mathrm{kV}$; capillary voltage, $11 \mathrm{~V}$; capillary temperature, $220{ }^{\circ} \mathrm{C}$; tube lens offset, $35 \mathrm{~V}$. Product ion spectra were acquired using an isolation width of $6 \mathrm{~m} / \mathrm{z}$ units ( $1 \mathrm{~m} / \mathrm{z}$ unit for the H/D exchange experiments), an activation Q of 0.250 and an activation time of 30 msec. The normalised collision energy was set to $50 \%$ and WideBand activation was switched on. Acquisitions were performed for twenty scans. Helium was used as the buffer gas. Data acquisition was performed using XCalibur version 1.2 (Thermo Fisher Scientific, San Jose, CA, USA).

Accurate mass measurements were performed using an Apex III FT-ICR mass spectrometer (Bruker, Billerica, MA, USA) equipped with a 4.7 Tesla actively shielded superconducting magnet, an Infinity cylindrical analyser cell and an Apollo ESI source. Positive ion ESI was performed by infusing the solutions directly into the source at a constant flow rate of

$3 \mu \mathrm{L} \mathrm{min}{ }^{-1}$. Nitrogen was used as the drying gas. The source conditions were; capillary voltage, $-4.5 \mathrm{kV}$; end plate voltage, $-3.8 \mathrm{kV}$; capillary exit voltage, $100 \mathrm{~V}$; skimmer $1,11 \mathrm{~V}$; skimmer 2, $6 \mathrm{~V}$; Offset, 1.25; Rf amplitude, $600 \mathrm{~Hz}$; dry gas temperature, $250{ }^{\circ} \mathrm{C}$; dry gas flow rate, 30 arbitrary units; nebulising gas pressure, 50 psi. The excitation amplitude was $3.7 \mathrm{~dB}$. The product ion spectra were acquired for eight scans using $1024 \mathrm{~K}$ data points. Argon was used as the collision gas. The MS/MS parameters were; corr sweep pulse length, 1000 Hsec; corr sweep attenuation, 37.5 dB; ejection safety belt, $3000 \mathrm{~Hz}$; user pulse length, 
$1000 \mu \mathrm{sec}$; ion activation pulse length, $250000 \mu \mathrm{sec}$; ion activation attenuation, $37.2 \mathrm{~dB}$; frequency offset from activation mass, - $500 \mathrm{~Hz}$; user delay length, $3 \mathrm{sec}$. The instrument was calibrated using the first-generation product ion spectrum of protonated reserpine. The

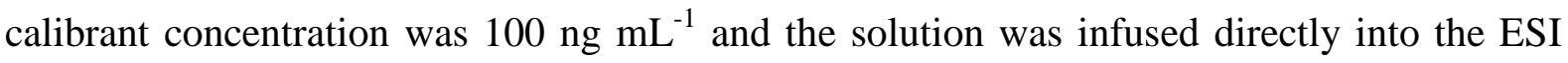
source at a constant flow rate of $3 \mu \mathrm{L} \mathrm{min}{ }^{-1}$. Data acquisition was performed using XMass version 7.0.8 (Bruker, Billerica, MA, USA).

\section{Results and discussion}

During a project to identify product ions and losses that facilitate rapid and definitive identification of pharmaceutical drug metabolites, the dissociation of a model S-oxidised metabolite, 3-dimethylaminomethyl-4-(4-methanesulfinyl-3-methyl-phenoxy)benzenesulfonamide (1) (Figure 1), was investigated. The first generation product ion spectrum of the protonated molecule is shown in Figure 2. It was hypothesised that the ion at $\mathrm{m} / \mathrm{z} 321$ was due to the loss of the methyl sulfoxide group. Thus, the dissociation was thought to be of potential use in metabolite identification; the $62 \mathrm{~m} / \mathrm{z}$ unit loss being assigned as methanethial, S-oxide $\left(\mathrm{CH}_{2} \mathrm{SO}\right)$. S-oxidation of the parent compound produces an ideal candidate for the loss of $62 \mathrm{~m} / \mathrm{z}$ units through the well-documented processes of chargeremote fragmentation ${ }^{12}$ and 1,3-proton shifts ${ }^{13}$ via four-centred rearrangements (Figure 3). This hypothesis was further attractive due to the absence of the same $62 \mathrm{~m} / \mathrm{z}$ unit loss from the parent compound (data not shown). Thus, it was postulated that the loss involved the oxygen atom. The dissociation was thought to be energetically favourable due to the conformance of the postulated product ion with the even-electron rule. ${ }^{14}$ However, the loss could not be confirmed by a change in the ${ }^{34} \mathrm{~S}$ isotope pattern for the product ion compared to the precursor ion due to the difficulties associated with observing the true isotope pattern using a QIT mass spectrometer. The overlapping of the A + 2 peak with the peak due to the lightest isotopes of the product ion at $\mathrm{m} / \mathrm{z} 323$ further precluded this approach to structural elucidation.

It was noted that the $62 \mathrm{~m} / \mathrm{z}$ unit loss involved none of the three exchangeable hydrogen atoms on the precursor ion. Thus, if the assignment of the loss was correct, the $\mathrm{m} / \mathrm{z}$ of the product ion would shift by $3 \mathrm{~m} / \mathrm{z}$ units to $\mathrm{m} / \mathrm{z} 324$ in the product ion spectrum of the fully exchanged, deuterated species (Figure 4). The absence of a peak at $\mathrm{m} / \mathrm{z} 324$ in the deuterated sample indicated that the initially posited loss was incorrect. Further, an additional peak was 
observed at $\mathrm{m} / \mathrm{z} 323$ in the product ion spectrum of the fully, exchanged deuterated species that was not seen for the protonated molecule. It should be noted that this ion was different to that seen at $m / z 323$ in the product ion spectrum of the protonated molecule, which was mass shifted to $\mathrm{m} / \mathrm{z} 325$ under deuterated conditions. The observation of an additional peak led to the deduction that two nominally isobaric product ions were formed through dissociation of the protonated species. The product ions only became mass resolved when the fully exchanged, deuterated molecule was fragmented due to the retention of different numbers of deuterium atoms by the two product ions. Thus, the H/D exchange experiment showed that two losses of $62 \mathrm{~m} / \mathrm{z}$ units took place, neither of which was that initially postulated. The loss of all of the deuterium atoms to form the product ion at $\mathrm{m} / \mathrm{z} 321$ indicated that the primary amine group had been lost during the dissociation. This was because exchange of two hydrogen atoms for deuterium labels would have occurred at this functional group. The peak at $\mathrm{m} / \mathrm{z} 323$, formed through a loss involving one deuterium atom, indicated that this product ion retained the primary amine. Further, it suggested that the ionising deuteron was lost during the dissociation, and hence the loss included a site of ionisation.

Further experimentation was undertaken to characterise the two losses of $62 \mathrm{~m} / \mathrm{z}$ units. To this end, the first generation product ion spectrum of a deuterium labelled analogue, 3-dimethyl${ }^{2} \mathrm{H}_{6}$-aminomethyl-4-(4-methanesulfinyl-3-methyl-phenoxy)-benzenesulfonamide (2) (Figure 5), was acquired (Figure 6). In the mass range of interest, the product ion spectrum resembled that of protonated 1 with only two peaks being observed. This indicated that the product ions were nominally isobaric again and neither product ion retained the six deuterium labels. Thus, both losses of $62 \mathrm{~m} / \mathrm{z}$ units involved the tertiary amine group. The H/D exchange experiment had shown that one product ion also lost the primary amine group; thus the overall loss was postulated to be $\mathrm{C}_{2} \mathrm{H}_{10} \mathrm{~N}_{2}$. The same experiment had also shown that one product ion retained the primary amine and lost the ionising deuteron. From this information, it was deduced that the tertiary amine group was a site of protonation. Further, it suggested that the remaining loss of $17 \mathrm{~m} / \mathrm{z}$ units for the product ion that retained the primary amine group (62 $\mathrm{m} / \mathrm{z}$ units minus $45 \mathrm{~m} / \mathrm{z}$ units for the tertiary amine group plus the ionising proton) was likely to be a hydroxyl radical; an overall proposed loss of $\mathrm{C}_{2} \mathrm{H}_{8} \mathrm{NO}^{\circ}$.

To confirm the hypothesised losses, accurate mass measurements were performed using Fourier transform-ion cyclotron resonance (FT-ICR) mass spectrometry. Figure 7 shows the first generation product ion spectrum of protonated $\mathbf{1}$ in the mass range of interest. The high 
resolving power of the FT-ICR mass spectrometer allowed mass resolution of the two nominally isobaric ions at $\mathrm{m} / \mathrm{z} 321$. The lowest error elemental formulae, given the precursor ion formula, were consistent with those postulated and confirmed that the two losses of $62 \mathrm{~m} / \mathrm{z}$ units were $\mathrm{C}_{2} \mathrm{H}_{10} \mathrm{~N}_{2}$ and $\mathrm{C}_{2} \mathrm{H}_{8} \mathrm{NO}^{\circ}$.

The verification of the elemental composition of the losses allowed postulation of dissociation mechanisms leading to the decomposition of the precursor ion. The ion with an elemental formula of $\mathrm{C}_{15} \mathrm{H}_{13} \mathrm{O}_{4} \mathrm{~S}_{2}{ }^{+}$(3) had a ring plus double bond equivalents value of 9.5, confirming that it was an even-electron ion. ${ }^{15}$ Because of the deficiency of this value by 0.5 due to the addition of a proton to the molecule ${ }^{16}$, the number of rings plus double bond equivalents in the product ion must have been 10. Figure 8 shows a proposed mechanism leading to the formation of a product ion which satisfies this value; the double bonds of the sulfone group are discounted due to sulfur not being in its lowest valence state. ${ }^{15}$ Protonation is postulated on the primary amine to facilitate the loss of the two exchangeable hydrogen atoms as part of a molecule of ammonia. The posited product ion structure is predominately planar as evinced by the molecular model in Figure 9. This is likely to provide the driving force for the energetic rearrangement involving a high energy proton shift leading to the loss of dimethylmine. ${ }^{17}$ The planar structure will allow easy delocalisation of the charge, making the formation of the product ion thermodynamically favourable.

The ion with an elemental formula of $\mathrm{C}_{15} \mathrm{H}_{15} \mathrm{NO}_{3} \mathrm{~S}_{2}{ }^{+\bullet}$ (4) had a ring plus double bond equivalents value of 10 i.e. an odd-electron species. ${ }^{15}$ Figure 10 shows a proposed mechanism to form a radical product ion structure that is consistent with this value. Again, the posited product ion structure is predominately planar (Figure 11), thus allowing facile delocalisation of the charge, and also the radical site. This is particularly important due to the energetically unfavourable formation of a radical product ion from an even electron precursor. ${ }^{18}$ However, the resonance stabilisation across the two aromatic rings, and the sulfone and sulfoxide functionalities, is likely to facilitate this dissociation.

Both proposed dissociation mechanisms involve the loss of dimethylamine. However, the pathways leading to this loss differ for the two product ions. The formation of $\mathbf{3}$ is postulated to involve the loss of dimethylamine facilitated by the shift of a non-exchangeable hydrogen atom. On the other hand, the formation of $\mathbf{4}$ proceeds via protonation of the tertiary amine followed by a charge-directed inductive cleavage of the carbon-nitrogen bond. Thus, an 
exchangeable hydrogen atom i.e. the ionising proton (deuteron), is proposed to facilitate the loss of dimethylamine in this instance. The loss of dimethylamine was also observed in the first generation product ion spectrum of protonated 1 (Figure 2; product ion at $\mathrm{m} / \mathrm{z} 338$ ). The dissociation of the fully exchanged, deuterated species (Figure 4) showed that the loss increased by $1 \mathrm{~m} / \mathrm{z}$ unit to produce a peak at $\mathrm{m} / \mathrm{z} 340$, demonstrating the involvement of a single exchangeable hydrogen atom in the fragmentation. The protonated molecule can not be spatial arranged such that the two exchangeable hydrogen atoms on the primary amine can be in close proximity to the tertiary amine functionality to allow intramolecular transfer. Thus, they can be excluded as being the source of the exchangeable hydrogen atom involved in the loss of dimethylamine. Hence, the exchangeable hydrogen atom must be the ionising proton (deuteron). From the two mechanisms postulated for the losses of $62 \mathrm{~m} / \mathrm{z}$ units (Figures 8 and 10), only one involves protonation at the tertiary amine and the loss of dimethylamine facilitated by an exchangeable hydrogen atom i.e. the loss of $\mathrm{C}_{2} \mathrm{H}_{8} \mathrm{NO}$. Protonation at the primary amine is inferred for the loss of $\mathrm{C}_{2} \mathrm{H}_{10} \mathrm{~N}_{2}$ to facilitate expulsion of a molecule of ammonia from the precursor ion. This could not be confirmed using H/D exchange experiments because the loss of ammonia is not observed in the first generation product ion spectrum of protonated 1 (Figure 2). However, supportive evidence for the proposed mechanisms was obtained through acquisition of the second generation product ion spectrum of fully exchanged, deuterated 1 using the ion at $\mathrm{m} / \mathrm{z} 340$ as the precursor ion for the second stage of mass analysis (Figure 12). The dissociation of the fully exchanged, deuterated molecule, rather than the protonated molecule, was investigated so that the product ions would be mass resolved on the QIT mass spectrometer if they were both formed. In the mass range of interest, a product ion was observed at $\mathrm{m} / \mathrm{z} 323$, which suggested that $\mathbf{4}$ is formed through protonation at the tertiary amine, followed by the loss of dimethylamine and then expulsion of a hydroxyl radical. The mass difference of $17 \mathrm{~m} / \mathrm{z}$ units between the precursor ion for the second stage of mass analysis and the final product ion indicated that the loss of the hydroxyl radical involved a non-exchangeable hydrogen atom. If an exchangeable hydrogen atom was involved, under deuterated conditions the loss would become a deuterohydroxyl radical and the mass loss would increase to $18 \mathrm{~m} / \mathrm{z}$ units. The observations were consistent with the proposed mechanism for the dissociation (Figure 10). No peak was observed at $m / z$ 321, which indicated that a second dissociation pathway exists for the loss of dimethylamine. This provided supportive evidence for a loss of dimethylamine facilitated by the shift of a non-exchangeable hydrogen atom, which is consistent with the proposed mechanism (Figure 8). The absence of the loss of ammonia and dimethylamine facilitated by 
a non-exchangeable hydrogen atom in the first generation product ion spectrum of protonated 1 suggested that the dissociation was concerted, or at least the intermediates underwent rapid dissociation to the final product ion. Further, the H/D exchange experiments suggested that $\mathbf{1}$ had more than one site of protonation in the gas-phase because the loss of the tertiary amine group was not via protonation at that site in the formation of $\mathbf{3}$. Thus, it was plausible that the molecule could protonate at the primary amine, further supporting the mechanism proposal.

The discussed dissociation has implications for structural elucidation using CID product ion spectra, particularly pharmaceutical drug metabolite identification studies. The structure of a metabolite must be deduced from its product ion spectrum. From the low resolution product ion spectrum of protonated $\mathbf{1}, S$-oxidation is likely to be proposed based upon the initial assignment of the $62 \mathrm{~m} / \mathrm{z}$ units loss as methanethial, $S$-oxide via a four-centred rearrangement. In this case, the proposal of S-oxidation would have been correct, but the assignments of ions leading to this proposal would have been incorrect. If this were an unknown metabolite, oxidation of the parent compound could have occurred, for example, through aromatic hydroxylation of the 1-methyl-2-methylsulfanyl-benzene ring and potentially not affected either of the actual losses of $62 \mathrm{~m} / \mathrm{z}$ units that took place. The attractive nature of $S$-oxidation facilitating the loss of methanethial, $S$-oxide via a fourcentred rearrangement may lead to mis-assignment of the ions in the CID spectrum. Hence time and money may be spent synthesising analogues and/or homologues to protect the methyl sulfide functionality of the parent compound only to discover that the hypothesised site of oxidation is incorrect. The discussed example highlights the possibility of unexpected rearrangements under CID conditions, which could easily lead to incorrect assignment of ion structure, and hence sites of metabolism in drug discovery studies. A simple and cost effective approach to reduce the likelihood of this occurrence is the use of H/D exchange experiments, which provide a straightforward method of introducing isotopic labels into the compound. Thus, dissociations proposed to involve exchangeable hydrogen atoms can be verified. Further, employment of mass spectrometers capable of high resolution, even when coupled to fast chromatography as utilised in metabolite identification studies, would also reduce the possibility of incorrect ion structure assignment. The study has also been of relevance to the understanding of fragmentation under CID conditions. The actual dissociations involved more complex rearrangements than that initially proposed, and also the energetically unfavourable formation of a radical product ion. However, the final shape of the product ion appears to have a bearing on the dissociation pathway followed. The initially 
proposed product ion structure formed through the loss of methanethial, S-oxide has free rotation about the ether linkage. The product ions actually formed are posited to be predominately planar, thus allowing easier delocalisation of the charge. This may suggest that the energetic favourability of the product ion structure can outweigh the facileness of the rearrangement and provide the driving force for dissociation; suggesting less dependence on the kinetics of the fragmentation under CID conditions compared to electron ionisation (EI) conditions. ${ }^{1}$ Furthermore, the study indicated that H/D exchange experiments can be useful in determining sites of gas-phase protonation under ESI-CID conditions, which has been demonstrated in a previous study ${ }^{19}$, thus allowing rationalisation of dissociation mechanisms.

\section{Conclusions}

The experimental investigation of the dissociation of a model $S$-oxidised metabolite has been presented. The ion at $\mathrm{m} / \mathrm{z} 321$ observed in the low resolution CID product ion spectrum was initially assigned as being formed through the loss of methanethial, $S$-oxide from the protonated molecule. It was thought that this loss could be used to assign the site of metabolism as the sulfur of the methyl sulfide group. Subsequent deuterium labelling experiments and accurate mass measurements acquired using a high resolution CID product ion spectrum proved that the initial assignment was incorrect. Instead, two unexpected rearrangements lead to the formation of nominally isobaric ions. Only through the use of H/D exchange experiments and high resolution mass spectrometry was it possible to mass resolve these species. Mechanisms consistent with the experimental findings were postulated and supportive evidence for the proposals was obtained through the acquisition of a second generation product ion spectrum. The proposed planar product ion structures were concluded to provide the driving force for the rearrangements, particularly in the formation of an energetically unfavourable radical cation. The study was of relevance to pharmaceutical drug metabolite identification because it demonstrated that a rigorous analytical approach is required to ensure that incorrect assignment of the sites of metabolism of novel chemical entities is precluded. A greater understanding of fragmentation under CID conditions was also obtained, with the shape of the product ion apparently offsetting the complexity of the rearrangement required to form it. Further, the data verified the usefulness of H/D exchange experiments in determining sites of protonation under ESI-CID conditions. 


\section{Acknowledgements}

This work was financially supported by the UK Biotechnology and Biological Research Council (BBSRC grant number BBS/S/N/2006/13093) and Pfizer Global Research and Development. The authors wish to thank Dr. Katerina Klagkou for helpful discussions relating to the dissociation mechanisms. 


\section{Figures}

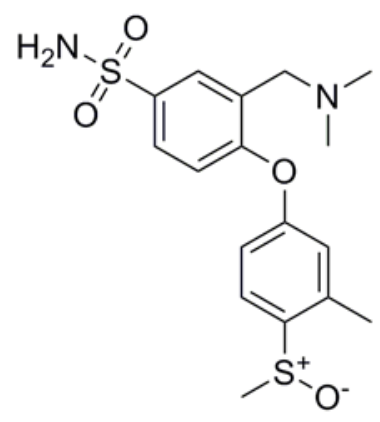

Figure 1. Molecular structure of 3-dimethylaminomethyl-4-(4-methanesulfinyl-3-methyl-phenoxy)-benzenesulfonamide (1) 


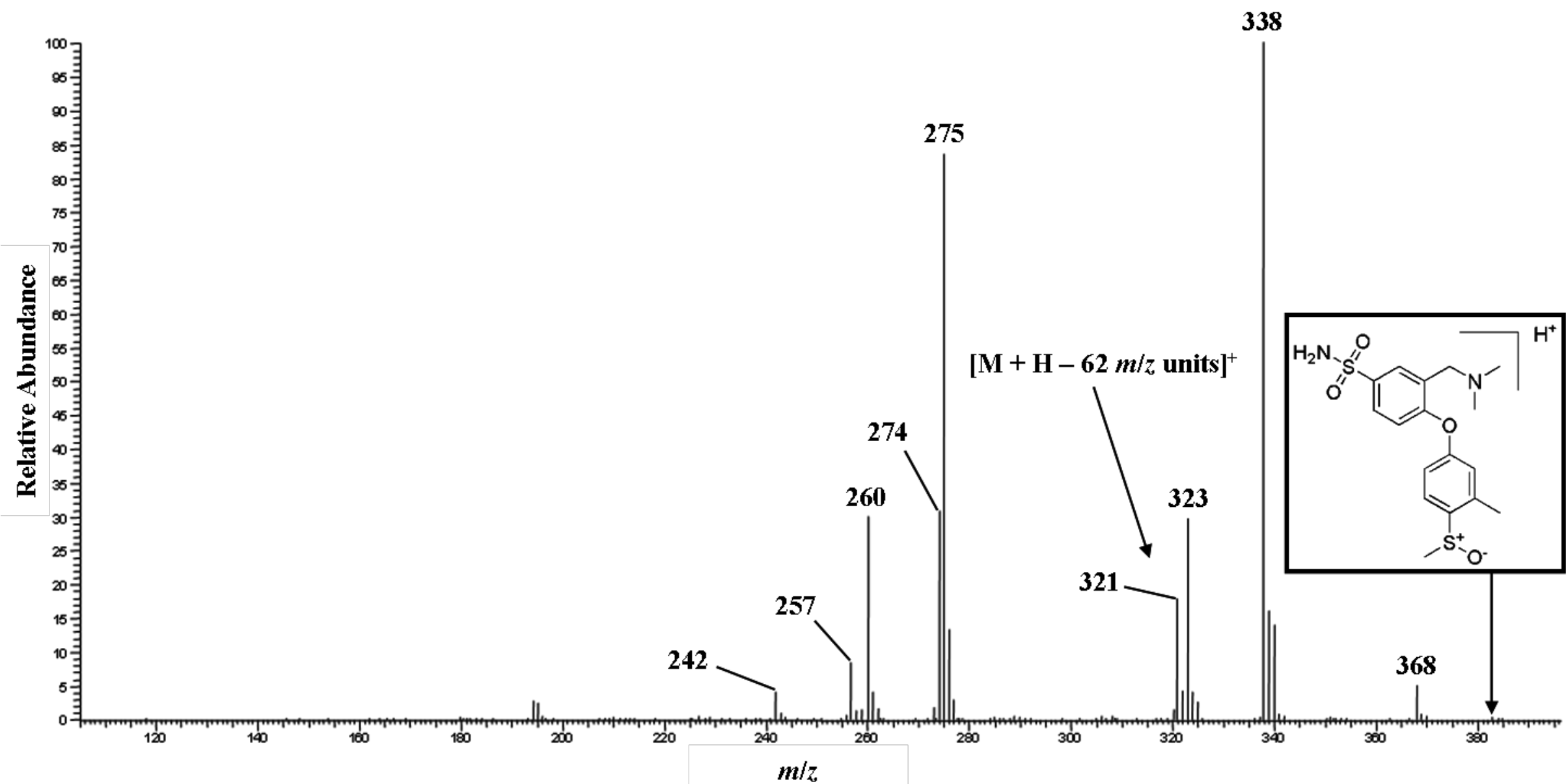

Figure 2. First generation product ion spectrum of protonated $\mathbf{1}$ acquired using a LCQ Classic QIT mass spectrometer with WideBand activation on 

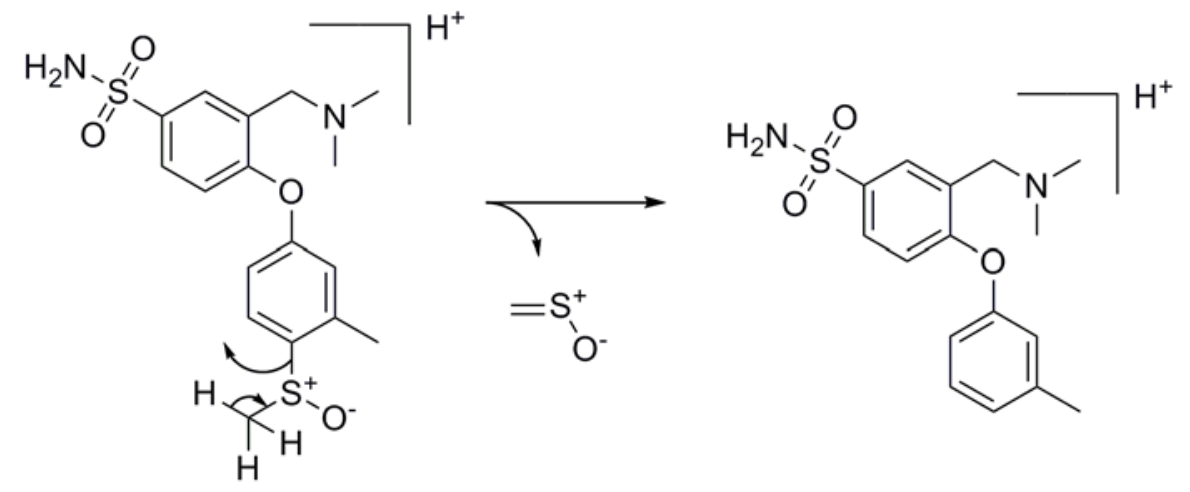

Figure 3. Proposed mechanism for the loss of methanethial, S-oxide from protonated $\mathbf{1}$ via a four-centred rearrangement 


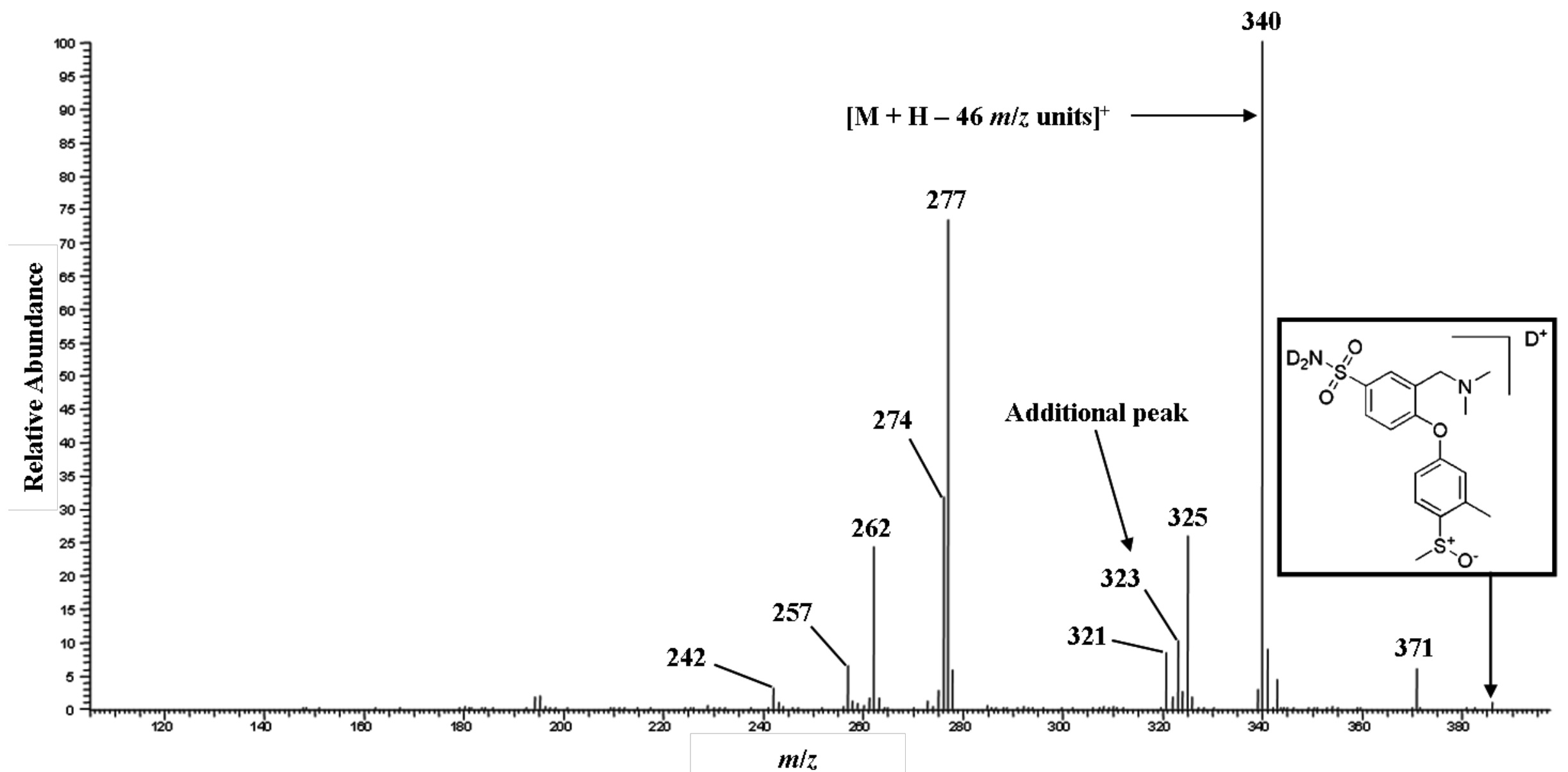

Figure 4. First generation product ion spectrum of fully exchanged, deuterated 1 acquired using a LCQ Classic QIT mass spectrometer with WideBand activation on 


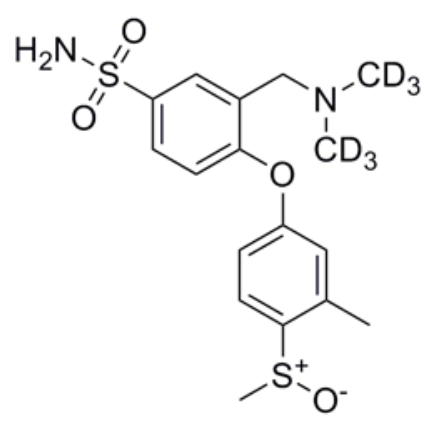

Figure 5. Molecular structure of 3-dimethyl- ${ }^{2} \mathrm{H}_{6}$-aminomethyl-4-(4-methanesulfinyl-3-methyl-phenoxy)-benzenesulfonamide (2) 


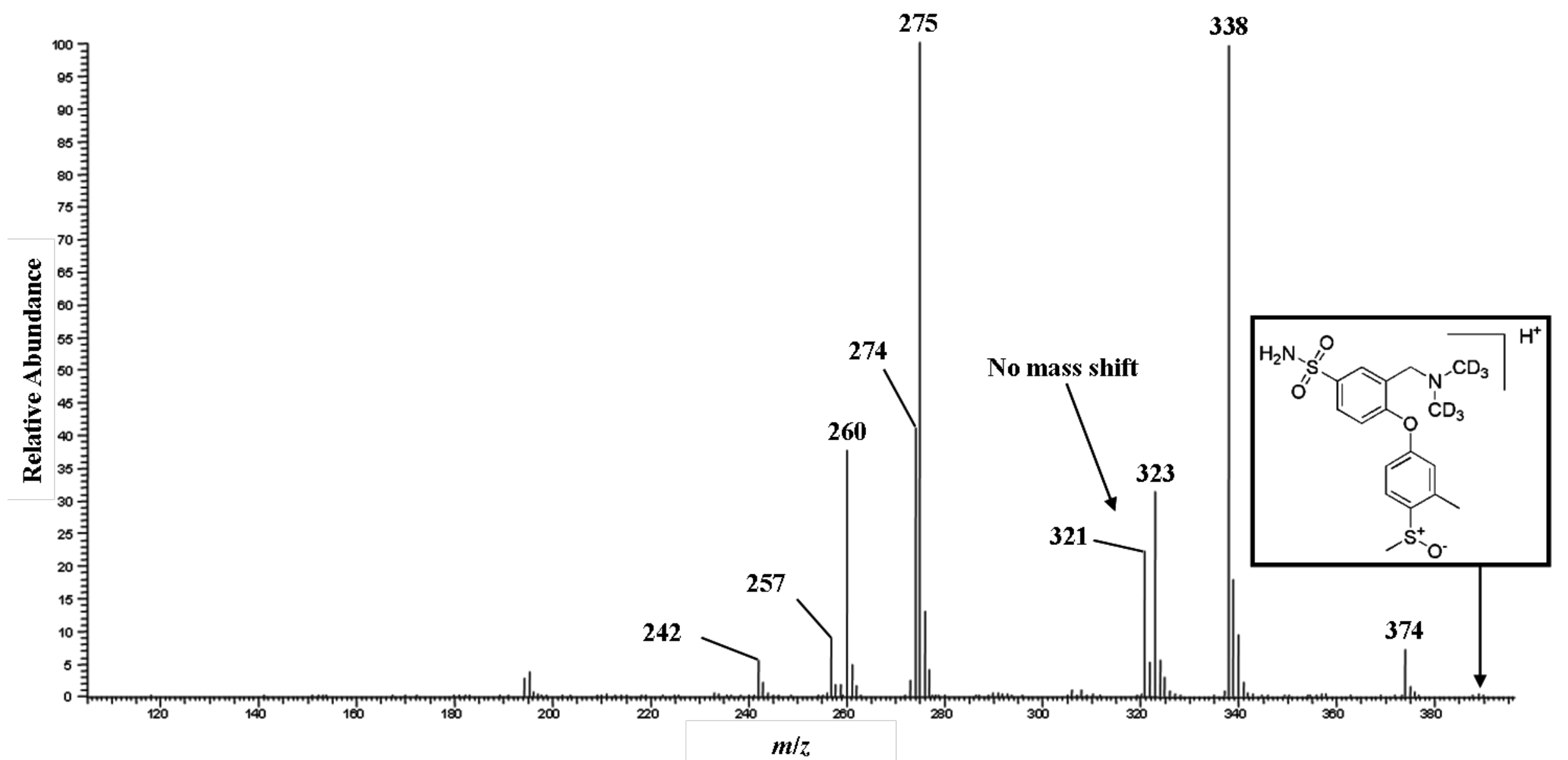

Figure 6. First generation product ion spectrum of protonated 2 acquired using a LCQ Classic QIT mass spectrometer with WideBand activation on 


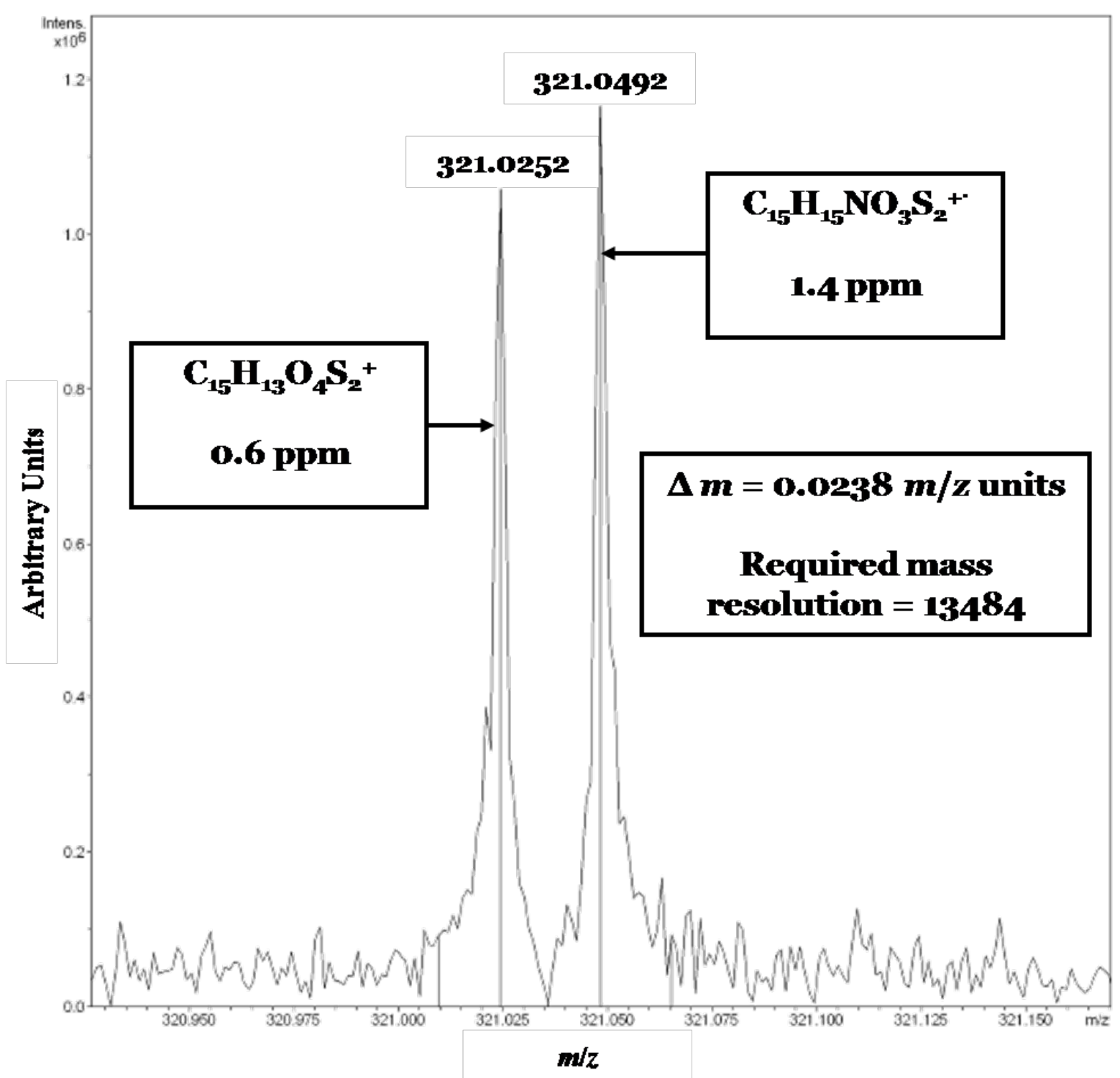

Figure 7. First generation product ion spectrum of protonated $\mathbf{1}$ acquired using an Apex III FT-ICR mass spectrometer 

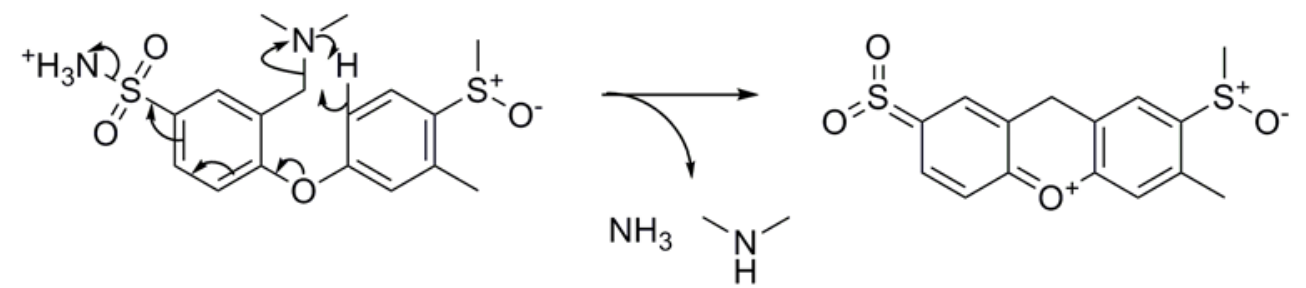

Figure 8. Proposed mechanism for the formation of 3 through the loss of $\mathrm{C}_{2} \mathrm{H}_{10} \mathrm{~N}_{2}$ as molecules of ammonia and dimethylamine from protonated 1 

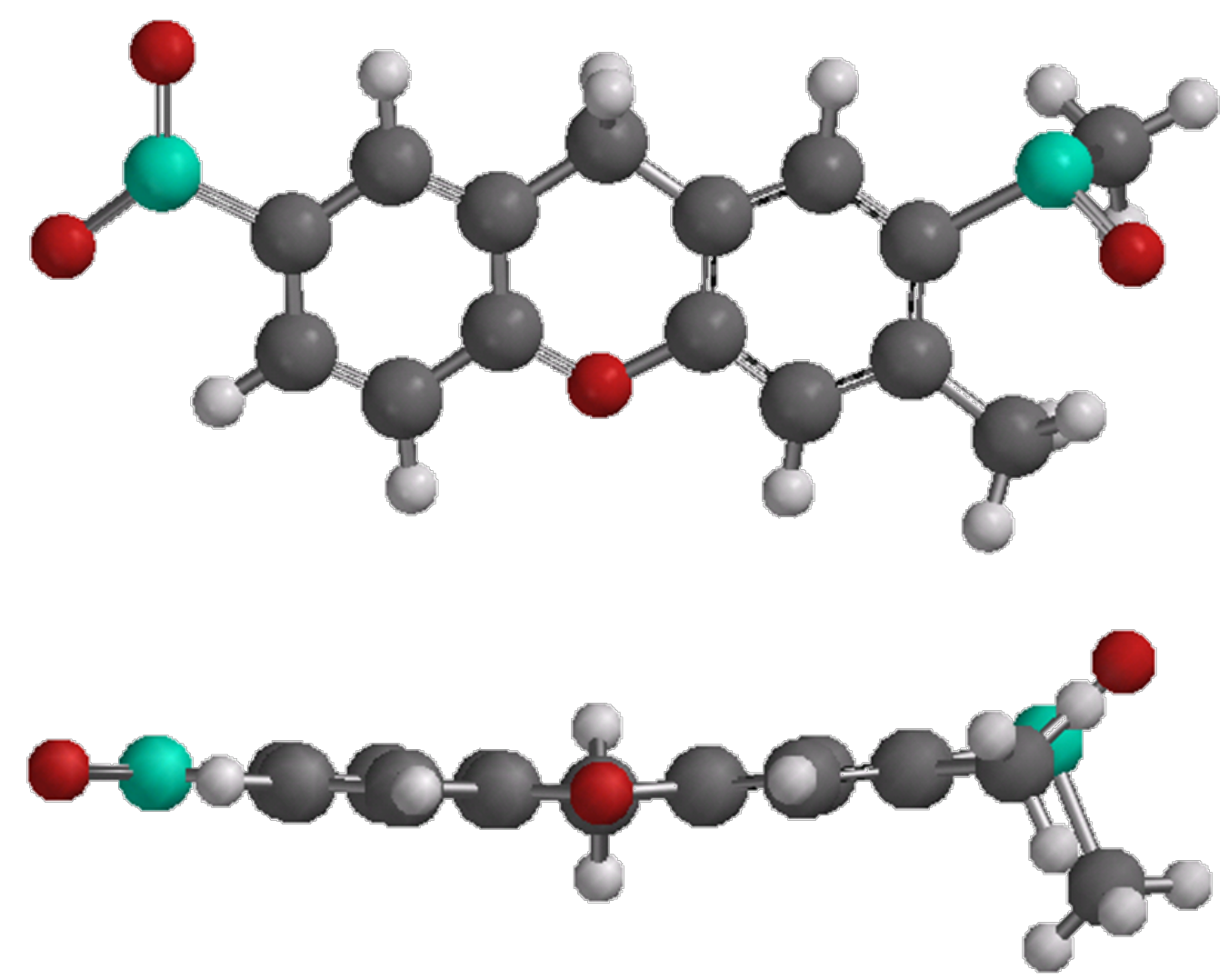

Figure 9. Molecular models of $\mathbf{3}$ demonstrating the predominately planar structure of the product ion 


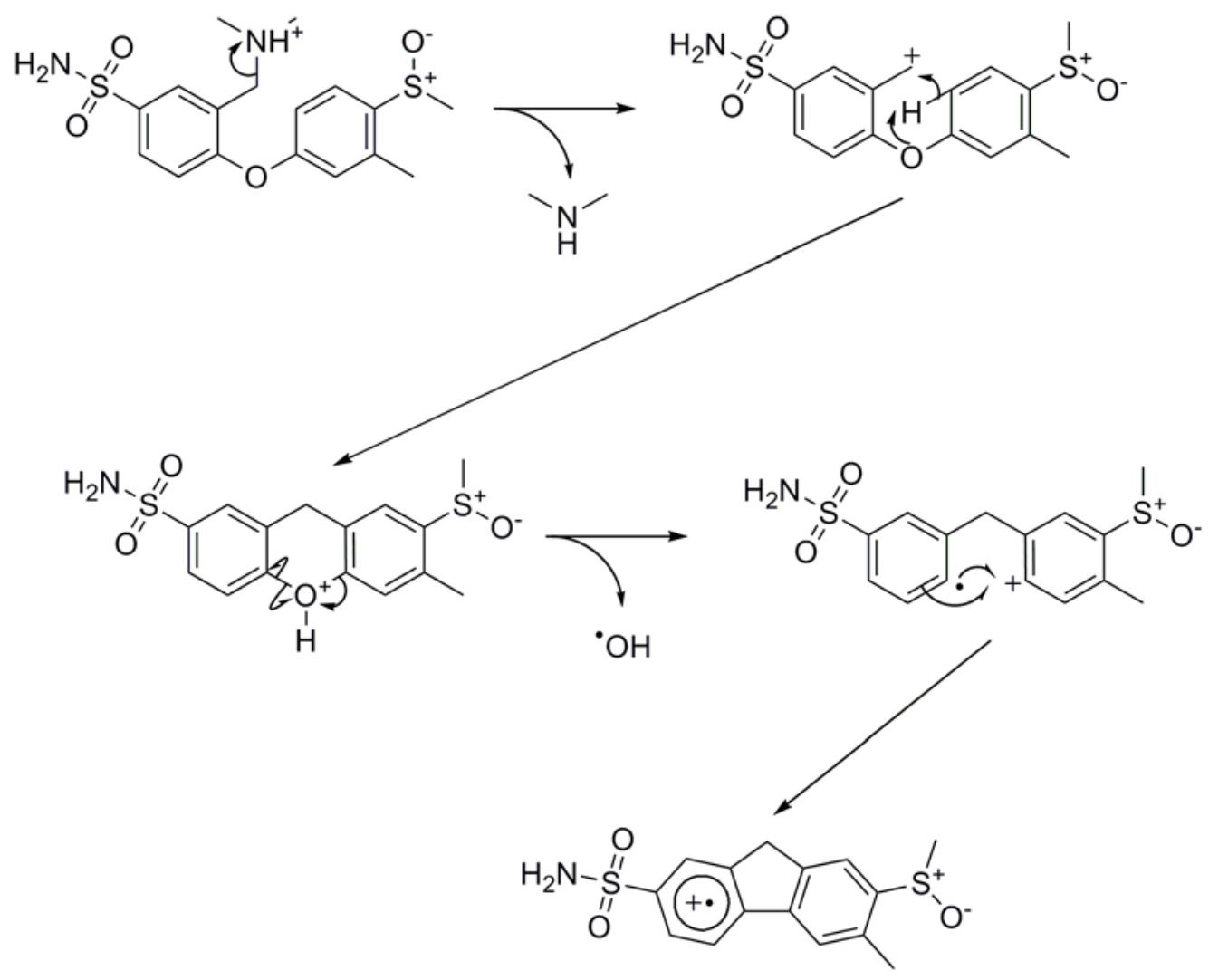

Figure 10. Proposed mechanism for the formation of 4 through the loss of $\mathrm{C}_{2} \mathrm{H}_{8} \mathrm{NO}{ }^{\bullet}$ as a molecule dimethylamine and a hydroxyl radical from protonated 1 

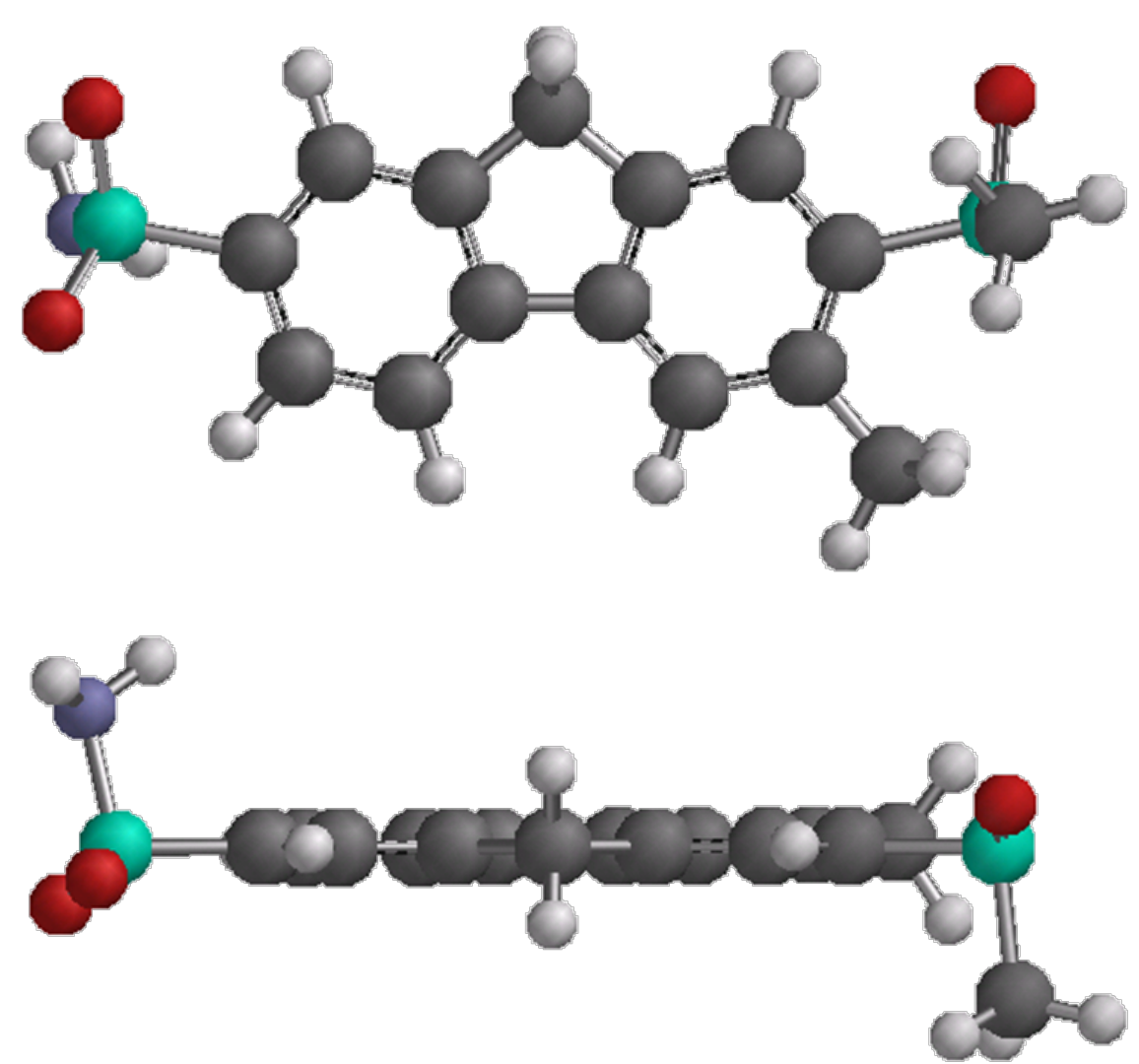

Figure 11. Molecular models of $\mathbf{4}$ demonstrating the predominately planar structure of the product ion 


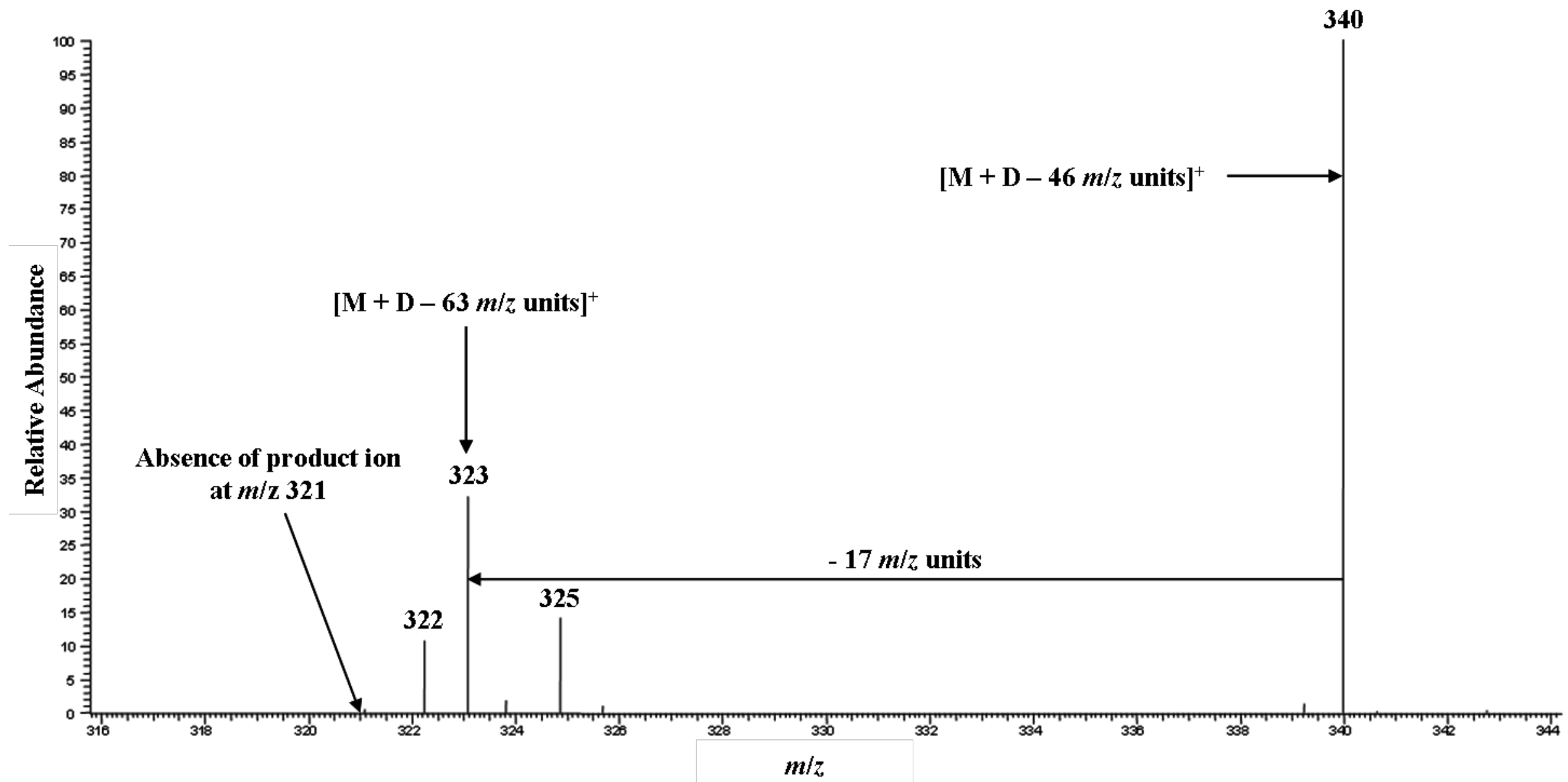

Figure 12. Second generation product ion spectrum of fully exchanged, deuterated $\mathbf{1}$ using the ion at $\mathrm{m} / \mathrm{z} 340$ as the precursor ion for the second stage of mass analysis acquired using a LCQ Classic QIT mass spectrometer with WideBand activation on 


\section{References}

1. C. Prakash, C. L. Shaffer and A. Nedderman, Mass Spectrom. Rev., 2007, 26, 340369.

2. R. Ramanathan, D. L. McKenzie, M. Tugnait and K. Siebenaler, J. Pharm. Biomed. Anal., 2002, 28, 945-951.

3. A. Fura, Drug Discov. Today, 2006, 11, 133-142.

4. S.-W. Myung, H.-Y. Kim, H.-K. Min, D.-H. Kim, M. Kim, H.-W. Choo, H. S. Lee, J.-K. Kim and C. I. Hong, Rapid Commun. Mass Spectrom., 2002, 16, 2048-2053.

5. $\quad$ S. Ma and R. Subramanian, J. Mass Spectrom., 2006, 41, 1121-1139.

6. $\quad$ K. Biemann, J. Am. Soc. Mass Spectrom., 2002, 13, 1254-1272.

7. P. Wright, A. Alex, D. Gibson, R. Jones and P. Macrae, Rapid Commun. Mass Spectrom., 2005, 19, 2005-2014.

8. P. R. Tiller, C. Raab and C. E. C. A. Hop, J. Mass Spectrom., 2001, 36, 344-345.

9. A. Cartoni, M. Altamura, F. Animati, G. Balacco, R. Cosi, A. Ettorre, A. Madami and A. Triolo, J. Mass Spectrom., 2002, 37, 1258-1265.

10. R. V. Vachet, B. M. Bishop, B. W. Erickson and G. L. Glish, J. Am. Chem. Soc., 1997, 119, 5481-5488.

11. A. G. Craig and S. W. Taylor, J. Am. Soc. Mass Spectrom., 2001, 12, 470-474.

12. C. Cheng and M. L. Gross, Mass Spectrom. Rev., 2000, 19, 398-420.

13. C. E. Hudson and D. J. McAdoo, J. Am. Soc. Mass Spectrom., 2004, 15, 972-981.

14. M. Karni and A. Mandelbaum, Org. Mass Spectrom., 1980, 15, 53-64.

15. F. W. McLafferty and F. Turecek, Interpretation of Mass Spectra, 4th Edn., University Science Books, Sausalito, 1993.

16. V. Pellegrin, J. Chem. Educ., 1983, 60, 626-633.

17. J. T. Bursey, M. M. Bursey and D. G. I. Kingston, Chem. Rev., 1973, 73, 191-234.

18. R. G. Cooks, Org. Mass Spectrom., 1969, 2, 481-519.

19. S. W. Holman, P. Wright and G. J. Langley, Rapid Commun. Mass Spectrom., 2008, 22, 2355-2365. 